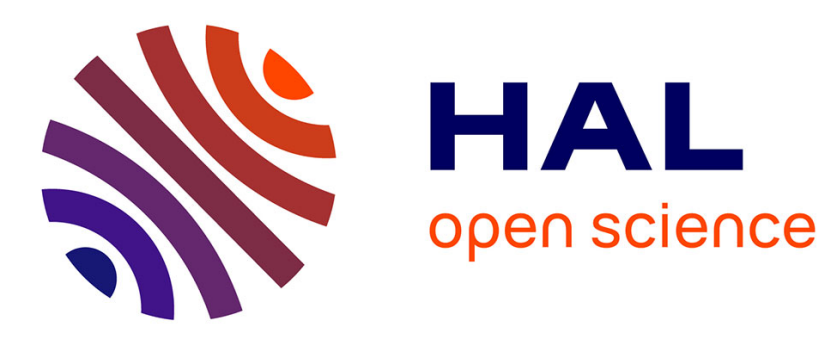

\title{
Nondestructive testing of the delaminated interface between two materials
}

\author{
Fioralba Cakoni, Irene de Teresa, Houssem Haddar, Peter Monk
}

\section{To cite this version:}

Fioralba Cakoni, Irene de Teresa, Houssem Haddar, Peter Monk. Nondestructive testing of the delaminated interface between two materials. SIAM Journal on Applied Mathematics, 2016, 76 (6), pp. 2306-2332. 10.1137/16M1064167 . hal-01373251

\section{HAL Id: hal-01373251 \\ https://inria.hal.science/hal-01373251}

Submitted on 28 Sep 2016

HAL is a multi-disciplinary open access archive for the deposit and dissemination of scientific research documents, whether they are published or not. The documents may come from teaching and research institutions in France or abroad, or from public or private research centers.
L'archive ouverte pluridisciplinaire HAL, est destinée au dépôt et à la diffusion de documents scientifiques de niveau recherche, publiés ou non, émanant des établissements d'enseignement et de recherche français ou étrangers, des laboratoires publics ou privés. 


\title{
NONDESTRUCTIVE TESTING OF THE DELAMINATED INTERFACE BETWEEN TWO MATERIALS
}

\author{
FIORALBA CAKONI*, IRENE DE TERESA ${ }^{\dagger}$, HOUSSEM HADDAR $^{\ddagger}$, AND PETER MONK ${ }^{\S}$
}

\begin{abstract}
We consider the problem of detecting if two materials that should be in contact have separated or delaminated. The goal is to find an acoustic technique to detect the delamination. We model the delamination as a thin opening between two materials of different acoustic properties, and using asymptotic techniques we derive a asymptotic model where the delaminated region is replaced by jump conditions on the acoustic field and flux. The asymptotic model has potential singularities due to the edges of the delaminated region, and we show that the forward problem is well posed for a large class of possible delaminations. We then design a special Linear Sampling Method (LSM) for detecting the shape of the delamination assuming that the background, undamaged, state is known. Finally we show, by numerical experiments, that our LSM can indeed determine the shape of delaminated regions.
\end{abstract}

Key words. Nondestructive testing, delamination, cracks, inverse problem, linear sampling method, asymptotic methods.

AMS subject classifications. 35R30, 35Q60, 35J40, 78A25.

1. Introduction. Delamination of two materials occurs when one material becomes partially detached from the other. This occurs in composite structures [11], concrete [30] and other engineering applications (e.g. [31, 22]). In this paper we will develop an inverse scattering approach to the detection of delamination using acoustic waves. We consider two materials that should have a coincident boundary (in the undamaged or background state) and we wish to detect if there is a part of the common boundary where the two materials have separated. In particular we want to determine the size and position of the delamination.

More precisely, we denote by $\Omega \subset \mathbb{R}^{m}, m=2,3$ the support of the inhomogeneity to be tested which in absence of delamination is composed of two different materials adjacent to one another with constitutive material properties $\mu_{+}, n_{+}$and $\mu_{-}, n_{-}$. We denote their bounded support by $\Omega_{-}$and $\Omega_{+}$, respectively, and the shared interface by $\Gamma:=\partial \Omega_{-}$(i.e. $\Omega=\Omega_{-} \cup \Omega_{+}$). Both the outer boundary $\partial \Omega_{+}$of the domain $\Omega_{+}$and the boundary $\partial \Omega_{-}$of the simply connected domain $\Omega_{-}$are assumed to be piece-wise smooth, unless mentioned otherwise, and $\nu$ denotes the unit normal always oriented outwards to the region bounded by the curve. For simplicity we let $\Omega_{\text {ext }}:=\mathbb{R}^{m} \backslash \bar{\Omega}$. Furthermore, we assume that along a part of the interface, denoted here by $\Gamma_{0} \subset \Gamma$, these two materials have detached (delaminated) and we model this fact with the appearance of an opening with support $\Omega_{0}$ and material properties $\mu_{0}, n_{0}$ (see Fig. 1). Note that $\Gamma_{0}=\Omega_{0} \cap \Gamma$. The material properties (possibly complex valued) in each of the domains are assumed to be smooth, i.e. $\mu_{+}, n_{+} \in C^{1}\left(\Omega_{+}\right), \mu_{-}, n_{-} \in C^{1}\left(\Omega_{-}\right)$and $\mu_{0}, n_{0} \in C^{1}\left(\Omega_{0}\right)$ (however note that across the interfaces there are discontinuities in the material properties).

Assuming now that the incident field and the other fields in the problem are time harmonic (i.e. the time dependent incident field is of the form $\Re\left(u^{i}(x) e^{i \omega t}\right)$ where $\omega$ is the angular frequency), then the total field $u^{e x t}=u^{s}+u^{i}$ in $\Omega_{\text {ext }}$, where $u^{s}$ is

\footnotetext{
* Mathematics, Rutgers University Piscataway, NJ 08854-8019, USA (fiora.cakoni@rutgers.edu)

$\dagger$ Mathematical Sciences, University of Delaware, Newark, Delaware 19716, USA (irene@udel.edu)

${ }_{\ddagger}^{\ddagger}$ INRIA, CMAP, Ecole polytechnique, Université Paris Saclay, Route de Saclay, 91128 Palaiseau, France (haddar@cmap.polytechnique.fr)

$\S$ Mathematical Sciences, University of Delaware, Newark, Delaware 19716, USA (monk@udel.edu).
} 


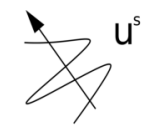

$\Omega_{\mathrm{ext}}$

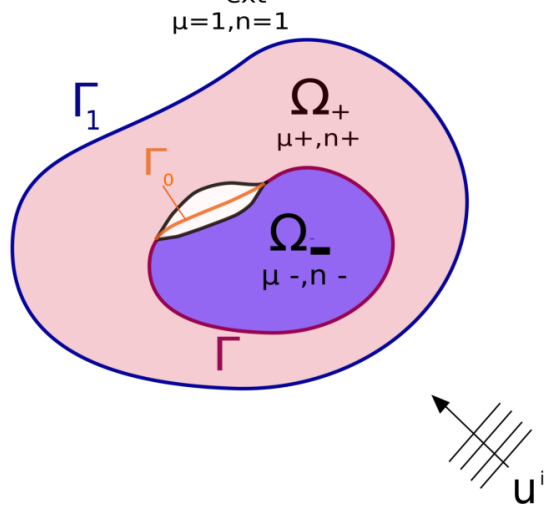

FIG. 1. Layered media with a thin delamination at the interface of two layers $\Omega_{-}$and $\Omega_{+}$. The opening $\Omega_{0}$, with coefficients $\mu_{0}, n_{0}$ is shown as the white region.

the scattered field, and the fields $u^{+}, u^{-}$and $U$ inside $\Omega_{+}, \Omega_{-}$and $\Omega_{0}$, respectively, satisfy

$$
\begin{aligned}
& \Delta u^{e x t}+k^{2} u^{e x t}=0 \quad \text { in } \quad \Omega_{\mathrm{ext}} \\
& \nabla \cdot\left(\frac{1}{\mu_{+}} \nabla u^{+}\right)+k^{2} n_{+} u^{+}=0 \quad \text { in } \quad \Omega_{+} \\
& \nabla \cdot\left(\frac{1}{\mu_{-}} \nabla u^{-}\right)+k^{2} n_{-} u^{-}=0 \quad \text { in } \quad \Omega_{-} \\
& \nabla \cdot\left(\frac{1}{\mu_{0}} \nabla U\right)+k^{2} n_{0} U=0 \quad \text { in } \quad \Omega_{0} .
\end{aligned}
$$

Here the wave number $k=\omega / c_{\text {ext }}$ with $c_{\text {ext }}$ denoting the sound speed of the homogeneous background. Across the interfaces the fields on either side and their conormal derivatives are continuous, i.e.

$$
\begin{aligned}
& u^{e x t}=u^{+} \quad \text { and } \quad \frac{\partial u^{e x t}}{\partial \nu}=\frac{1}{\mu_{+}} \frac{\partial u^{+}}{\partial \nu} \quad \text { on } \Gamma_{1} \\
& u^{+}=u^{-} \quad \text { and } \quad \frac{1}{\mu_{+}} \frac{\partial u^{+}}{\partial \nu}=\frac{1}{\mu_{-}} \frac{\partial u^{-}}{\partial \nu} \quad \text { on } \Gamma \backslash \bar{\Gamma}_{0} \\
& U=u^{+} \quad \text { and } \quad \frac{1}{\mu_{0}} \frac{\partial U}{\partial \nu}=\frac{1}{\mu_{+}} \frac{\partial u^{+}}{\partial \nu} \quad \text { on } \Gamma_{+} \\
& U=u^{-} \quad \text { and } \quad \frac{1}{\mu_{0}} \frac{\partial U}{\partial \nu}=\frac{1}{\mu_{-}} \frac{\partial u^{-}}{\partial \nu} \quad \text { on } \Gamma_{-} .
\end{aligned}
$$

Of course the scattered field $u^{s}$ satisfies the Sommerfeld radiation condition

$$
\lim _{r \rightarrow \infty} r^{\frac{m-1}{2}}\left(\frac{\partial u^{s}}{\partial r}-i k u^{s}\right)=0
$$

uniformly in $\hat{x}=x /|x|$, where $x \in \mathbb{R}^{m}$ and $r=|x|$. In this paper we consider plane waves as incident fields which are given by $u^{i}:=e^{i k x \cdot d}$ where the unit vector $d$ is 
the incident direction. Instead of plane waves, it is also possible to consider incident waves due to point sources located outside $\Omega$, in which case the obvious modifications need to be made in the formulation of the problem.

The goal of the this study is to propose and analyze a Linear Sampling Method (LSM) type scheme for detecting the delaminated region using remote measurements of acoustic waves scattered by the structure. In practice, the thickness of the opening is much smaller than both the interrogating wave length in free space $\lambda=\frac{2 \pi}{k}$ and the thickness of the layers of background material. This introduces an essential computational difficulty in the numerical solution of the forward problem as well as in the solution of the corresponding inverse problem. In the following section we take advantage of the small scale of the thickness and, using an asymptotic method from [7, 29], we derive an approximate model of the delaminated structure where the opening $\Omega_{0}$ is replaced by new jump relations for $u^{+}$and $u^{-}$across the delaminated part $\Gamma_{0}$ that account for the presence of the opening. This is undertaken in Section 2 using formal asymptotic methods. Before analyzing the model further, we then demonstrate numerically that the asymptotic model predicts correctly the acoustic field and far field pattern of the scattered field for a particular model scatterer incorporating a delamination of small positive maximum width.

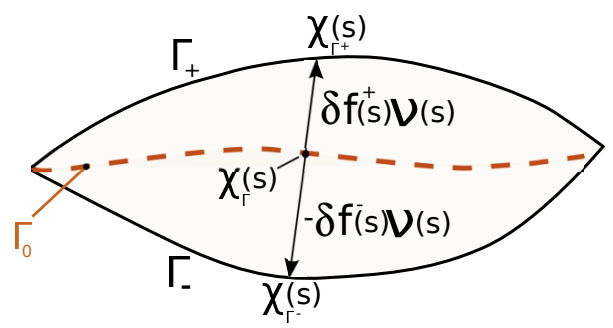

FIG. 2. Zoom of the thin delamination $\Omega_{0}$, and the parametrization of the boundaries $\Gamma_{-}$ and $\Gamma_{+}$. Here $\delta$ scales the width of the delamination and is assumed small compared to other characteristic dimensions of the problem.

Although there has been considerable work on the asymptotics of scattering from thin films (see for example $[5,2,3,4,6,24,20,7,29]$ ), the novelty of our reduced problem is that the delamination covers only a portion of the interface. The thickness of the delamination vanishes at its boundary and this introduces potential singularities into the asymptotic model. Therefore in Section 3 we analyze the forward reduced problem using an appropriate variational formulation and show that under reasonable conditions on the constitutive parameters and on the shape of the delamination the forward asymptotic model has a solution (indeed it is this variational scheme that was used to generate the finite element solution used in Section 2). Of course a thorough understanding of the forward model is also needed in our analysis of the inverse problem.

The inverse problem under study is precisely formulated in Section 4. We assume that the background or undamaged state is known, and then seek to determine the delaminated region $\Gamma_{0}$ using remote (far field) acoustic measurements. In preparation for the analysis of our scheme and to allow a simple calculation of the right hand side of the far field equation we then prove a new mixed reciprocity result for layered media. Next in Section 4.2 we give details of the LSM: in particular we seek to determine whether small artificial test arcs on the interface are within the delamination or in the undamaged region. This requires a suitable testing function for the LSM adapted to 
the delamination problem. We then prove the usual theorem for the LSM suggesting that an approximate solution of the far field equation can be used as an indicator function for the delamination.

Finally in Section 5 we test the inversion scheme on synthetic data for a special choice of the testing function from Section 4.2. In particular we show that our LSM can detect delamination even in the presence of noise on the data, and that multiple delaminated regions can be detected.

2. An approximate asymptotic model. In this section we assume $m=2$ and, focusing our attention on a neighborhood of the opening $\Omega_{0}$, use formal asymptotic analysis to derive an approximate model that takes into account the thin opening $\Omega_{0}$. To this end, we start by assuming that the portion $\Gamma_{0}$ of the boundary can be written in the form

$$
\Gamma_{0}:=\left\{\chi_{\Gamma}(s), \quad s \in[0, L]\right\}
$$

where $\chi_{\Gamma} \in C^{1}[0, L]$ is the counter-clockwise arc-length parametrization of $\Gamma_{0}$. If the curve $\Gamma_{0}$ is regular and $c(s)$ denotes its curvature at $\chi_{\Gamma}(s)$, then $0 \leq c_{m}:=$ $\max \{|c(s)|: s \in[0, L]\}$ is finite. Hence, in the neighborhood of $\Gamma_{0}$, one can define the curvilinear coordinates $(s, \eta) \in[0, L] \times\left(-\frac{1}{c_{m}}, \frac{1}{c_{m}}\right)$ by

$$
x=\chi_{\Gamma}(s)+\eta \nu(s)
$$

where we recall that $\nu$ is the unit normal vector on $\Gamma_{0}$ oriented outward to $\Omega_{-}$(and taking $\frac{1}{c_{m}}=\infty$ if $c_{m}=0$ ). Therefore, if the curvature of $\Gamma_{0}$ is small enough, both the outer and inner boundaries of $\Omega_{0}$, denoted here by $\Gamma_{+}$and $\Gamma_{-}$, can be written in this coordinate system as

$$
\Gamma_{+}=\left\{\chi_{\Gamma_{+}}(s):=\chi_{\Gamma}(s)+\delta f^{+}(s) \nu(s), \quad s \in[0, L]\right\}
$$

and

$$
\Gamma_{-}=\left\{\chi_{\Gamma_{-}}(s):=\chi_{\Gamma}(s)-\delta f^{-}(s) \nu(s), \quad s \in[0, L]\right\}
$$

Note that the function $\delta\left(f^{+}+f^{-}\right)(s)$ defined on $\Gamma_{0}$ describes the thickness of the delamination. Here $\delta$ is a small parameter (compared to both the wave length and the size of the domains involved), and $\max _{s \in[0, L]} f^{ \pm}(s)=1$ (see Figure 2).

In an open neighborhood of $\Omega_{0}$, we can now express the fields $U, u^{-}$, and $u^{+}$ in terms of the curvilinear variables $(s, \eta)$. Ignoring small neighborhoods of the tip points $s=0$ and $s=L$, since $\Omega_{0}$ plays here the role of a boundary layer, in order to transfer the small parameter $\delta$ from the geometry to the expression of the fields we make a stretching change of variables inside $\Omega_{0}$ defined by $\xi=\frac{\eta}{\delta}$. Hence, $\xi=\frac{\eta}{\delta}$ and $s$ are now the new coordinates inside $\Omega_{0}$. Next, following [7] and [29], we formally make the following ansatz for the fields $U$ and $u^{ \pm}$in an open neighborhood of $\Omega_{0}$

$$
U(s, \xi)=\sum_{j=0}^{\infty} \delta^{j} U_{j}(s, \xi)
$$

and

$$
u^{ \pm}(s, \eta)=\sum_{j=0}^{\infty} \delta^{j} u_{j}^{ \pm}(s, \eta),
$$


where neither $u_{j}^{ \pm}$nor $U_{j}$ depend on $\delta$ any longer. Furthermore, we expand each of the terms $u_{j}^{ \pm}(s, \eta)$ in a power series with respect to the normal direction coordinate $\eta$ around zero, i.e.

$$
u_{j}^{ \pm}(s, \eta)=u_{j}^{ \pm}(s, 0)+\eta \frac{\partial}{\partial \eta} u_{j}^{ \pm}(s, 0)+\frac{\eta^{2}}{2} \frac{\partial^{2}}{\partial \eta^{2}} u_{j}^{ \pm}(s, 0)+\ldots
$$

and after plugging in (2.2) we finally obtain the following expression for $u^{ \pm}(s, \eta)$,

$$
u^{ \pm}(s, \eta)=\sum_{j=0}^{\infty} \sum_{k=0}^{\infty} \delta^{j} \frac{\eta^{k}}{k !} \frac{\partial^{k}}{\partial \eta^{k}} u_{j}^{ \pm}(s, 0) .
$$

Now based on the ansatz (2.1) and (2.3), and using the equations along with the transmission conditions, we can formally obtain an approximate model for the field in the opening $\Omega_{0}$. For detailed calculations we refer the reader to [19] (see also [29]) and in the following we simply sketch the steps that lead to our approximate model.

2.1. The approximate transmission conditions. First we consider the expressions (2.1) and (2.3) which we substitute in (1.6), (1.7) and (1.8). To this end, starting with the Dirichlet part of the transmission conditions on $\Gamma_{ \pm}$, we can write

$$
U\left(s, \pm f^{ \pm}\right)=\sum_{j}^{\infty} \delta^{j} U_{j}\left(s, \pm f^{ \pm}\right)
$$

and

$$
u^{ \pm}\left(s, \pm \delta f^{ \pm}\right)=\sum_{j=0}^{\infty} \delta^{j} \sum_{k=0}^{j} \frac{( \pm 1)^{j-k}\left(f^{ \pm}\right)^{j-k}}{(j-k) !} \frac{\partial^{j-k}}{\partial \eta^{j-k}} u_{k}^{ \pm}(s, 0) .
$$

Then the Dirichlet part of the transmission condition can be directly computed by equating terms with the same powers of $\delta$. Doing so leads to

$$
U_{j}\left(s, \pm f^{ \pm}\right)=\sum_{k=0}^{j} \frac{( \pm 1)^{j-k}\left(f^{ \pm}\right)^{j-k}}{j-k !} \frac{\partial^{j-k}}{\partial \eta^{j-k}} u_{k}^{ \pm}(s, 0) \quad \text { for all } j=0,1,2, \ldots
$$

Next we deal with the Neumann part of the transmission conditions on $\Gamma_{ \pm}$. Unlike the Dirichlet part, the Neumann part of the transmission conditions is more delicate, because in order to compute the co-normal derivatives at $\Gamma_{ \pm}$, one has to take into account the expression in curvilinear coordinates of the normal vectors to those curves. To this end, as discussed in [7], the normal vectors $\nu^{ \pm}$on $\Gamma^{ \pm}$have the following expressions

$$
\nu^{ \pm}=\frac{1}{\left|\tau^{ \pm}\right|}\left(\left(1 \pm \delta f^{ \pm}\right) \nu \mp \delta \frac{d f^{ \pm}}{d s} \tau\right)
$$

where $\nu$ and $\tau$ are the outer unit normal vector and the unit tangential vector defined on $\Gamma_{0}$, respectively, whereas the tangent vectors $\tau^{ \pm}(s):=\frac{d}{d s} \chi_{\Gamma^{ \pm}}(s)$ to $\Gamma_{ \pm}$are not unit vectors. Next, in curvilinear coordinates the gradient operator takes the form

$$
\nabla u(x)=\frac{1}{1+\eta c} \frac{\partial u}{\partial s} \tau+\frac{\partial u}{\partial \eta} \nu
$$


where $c:=c(s)$ denotes the curvature function of $\Gamma_{0}$. Thus we now have all the ingredients to compute the Neumann part of the transmission conditions, and after straightforward but long calculations ([19]), the Neumann transmission conditions

$$
\left.\nu^{ \pm} \cdot \nabla u^{ \pm}\right|_{\Gamma^{ \pm}}=\left.\nu^{ \pm} \cdot \nabla U\right|_{\Gamma^{ \pm}},
$$

imply the following expression

$$
\begin{gathered}
\pm \frac{d f^{ \pm}}{d s}\left(\frac{1}{\mu_{0}} \frac{\partial U_{j-1}}{\partial s}\left(s, \pm f^{ \pm}\right)-\frac{1}{\mu_{ \pm}} \sum_{k=0}^{j-1} \frac{( \pm 1)^{j-k-1}\left(f^{ \pm}\right)^{j-k-1}}{(j-k-1) !} \frac{\partial^{j-k} u_{k}^{ \pm}}{\partial \eta^{j-k-1} \partial s}(s, 0)\right)= \\
(2.5) \quad\left(\frac{1}{\mu_{0}} \frac{\partial U_{j+1}}{\partial \xi}\left(s, \pm f^{ \pm}\right)-\frac{1}{\mu_{ \pm}} \sum_{k=0}^{j} \frac{( \pm 1)^{j-k}\left(f^{ \pm}\right)^{j-k}}{(j-k) !} \frac{\partial^{j-k+1} u_{k}^{ \pm}}{\partial \eta^{j-k+1}}(s, 0)\right) \\
\pm 2 f^{ \pm} c\left(\frac{1}{\mu_{0}} \frac{\partial U_{j}}{\partial \xi}\left(s, \pm f^{ \pm}\right)-\frac{1}{\mu_{ \pm}} \sum_{k=0}^{j-1} \frac{( \pm 1)^{j-k-1}\left(f^{ \pm}\right)^{j-k-1}}{(j-k-1) !} \frac{\partial^{j-k} u_{k}^{ \pm}}{\partial \eta^{j-k}}(s, 0)\right) \\
+c^{2}\left(f^{ \pm}\right)^{2}\left(\frac{1}{\mu_{0}} \frac{\partial U_{j-1}}{\partial \xi}\left(s, \pm f^{ \pm}\right)-\frac{1}{\mu_{ \pm}} \sum_{k=0}^{j-2} \frac{( \pm 1)^{j-k-2}\left(f^{ \pm}\right)^{j-k-2}}{(j-k-2) !} \frac{\partial^{j-k-1} u_{k}^{ \pm}}{\partial \eta^{j-k-1}}(s, 0)\right)
\end{gathered}
$$

for $j=-1,0,1,2, \ldots$, for all $s \in[0, L]$ and with the convention that $U_{l}=0$ and $u_{l}=0$ for $l<0$.

Next, we consider the partial differential equation satisfied by $U_{j}$. To this end, we write the differential operators in curvilinear coordinates and obtain

$$
\nabla \cdot\left(\frac{1}{\mu} \nabla w\right)=\frac{1}{(1+\eta c)} \frac{\partial}{\partial s}\left(\frac{1}{\mu} \frac{1}{(1+\eta c)} \frac{\partial w}{\partial s}\right)+\frac{1}{(1+\eta c)} \frac{\partial}{\partial \eta}\left(\frac{(1+\eta c)}{\mu} \frac{\partial w}{\partial \eta}\right) .
$$

Therefore, the equation satisfied by the field $U$ inside $\Omega_{0}$ in the new curvilinear coordinates is given by

$$
\frac{1}{(1+\delta \xi c)} \frac{\partial}{\partial s}\left(\frac{1}{\mu} \frac{1}{(1+\delta \xi c)} \frac{\partial U}{\partial s}\right)+\frac{1}{\delta} \frac{1}{(1+\delta \xi c)} \frac{\partial}{\partial \xi}\left(\frac{(1+\delta \xi c)}{\delta \mu} \frac{\partial U}{\partial \xi}\right)+k^{2} n_{0} U=0 .
$$

Now substituting the ansatz (2.1) and collecting the terms corresponding to same powers of $\delta$, we obtain

$$
\begin{gathered}
\frac{\partial}{\partial \xi}\left(\frac{1}{\mu_{0}} \frac{\partial}{\partial \xi}\right) U_{j}+\left(3 \xi c \frac{\partial}{\partial \xi}\left(\frac{1}{\mu_{0}} \frac{\partial}{\partial \xi}\right)+\frac{c}{\mu_{0}} \frac{\partial}{\partial \xi}\right) U_{j-1}+ \\
\left(\frac{\partial}{\partial s}\left(\frac{1}{\mu_{0}} \frac{\partial}{\partial s}\right)+3 \xi^{2} c^{2} \frac{\partial}{\partial \xi}\left(\frac{1}{\mu_{0}} \frac{\partial}{\partial \xi}\right)+\frac{2 c^{2} \xi}{\mu_{o}} \frac{\partial}{\partial \xi}+k^{2} n_{0}\right) U_{j-2}+ \\
\left(\xi c \frac{\partial}{\partial s}\left(\frac{1}{\mu_{0}} \frac{\partial}{\partial s}\right)+\xi^{3} c^{3} \frac{\partial}{\partial \xi}\left(\frac{1}{\mu_{0}} \frac{\partial}{\partial \xi}\right)+\frac{c^{3} \xi^{2}}{\mu_{o}} \frac{\partial}{\partial \xi}-\frac{\xi c^{\prime}}{\mu_{0}} \frac{\partial}{\partial s}+3 \xi c k^{2} n_{0}\right) U_{j-3}+ \\
+3 \xi^{2} c^{2} k^{2} n_{0} U_{j-4}+\xi^{3} c^{3} k^{2} n_{0} U_{j-5}=0
\end{gathered}
$$

for $j=0,1,2 \ldots$, and where again $c:=c(s)$ is the curvature of $\Gamma_{0}$ and conveying that $U_{l}=0$ for negative $l$.

The recursive relations for the transmission conditions (2.4) and (2.5), and the partial differential equation (2.6) of the three lowest order terms $U_{0}, U_{1}, U_{2}$ allow us to derive relations between the jumps and mean values of the outer fields $u_{0}$ and $u_{1}$ and 
their co-normal derivatives across $\Gamma_{0}$. In the following we summarize these relations (we refer the reader to ([19]) and ([29]) for details):

$$
\begin{gathered}
{\left[u_{0}\right]=0} \\
{\left[\frac{1}{\mu} \frac{\partial u_{0}}{\partial \nu}\right]=0} \\
{\left[u_{1}\right]=2\left\langle f\left(\mu_{0}-\mu\right)\right\rangle\left\langle\frac{1}{\mu} \frac{\partial u_{0}}{\partial \nu}\right\rangle} \\
{\left[\frac{1}{\mu} \frac{\partial u_{1}}{\partial \nu}\right]=2\left(\frac{\partial}{\partial s}\left(\left\langle f\left(\frac{1}{\mu}-\frac{1}{\mu_{0}}\right)\right\rangle \frac{\partial}{\partial s}\right)+k^{2}\left\langle f\left(n-n_{0}\right)\right\rangle\right)\left\langle u_{0}\right\rangle .}
\end{gathered}
$$

Here $\left[u_{i}\right]:=u_{i}^{+}(s, 0)-u_{i}^{-}(s, 0)$ and $\left\langle u_{i}\right\rangle:=\left(u_{i}^{+}(s, 0)+u_{i}^{-}(s, 0)\right) / 2, i=0,1$, are the point wise jump and average values of the outer fields on $\Gamma_{0}$. Analogously we use the symbols $\left[\frac{1}{\mu} \frac{\partial u_{i}}{\partial \nu}\right]$ and $\left\langle\frac{1}{\mu} \frac{\partial u_{i}}{\partial \nu}\right\rangle$ for the jump and average values of the conormal derivative on $\Gamma_{0}$, and similar definitions for the average values $\left\langle f\left(n-n_{0}\right)\right\rangle$, $\left\langle f\left(\frac{1}{\mu}-\frac{1}{\mu_{0}}\right)\right\rangle$, and $\left\langle f\left(\mu_{0}-\mu\right)\right\rangle$. Therefore, noting that $u^{ \pm}=u_{0}^{ \pm}+\delta u_{1}^{ \pm}+O\left(\delta^{2}\right)$, after dropping the $O\left(\delta^{2}\right)$-terms, we finally obtain the Approximate Transmission Conditions (ATCs) of the second order

$$
\begin{aligned}
{[u] } & =\alpha\left\langle\frac{1}{\mu} \frac{\partial u}{\partial \nu}\right\rangle \text { on } \Gamma_{0} \\
{\left[\frac{1}{\mu} \frac{\partial u}{\partial \nu}\right] } & =\left(-\frac{\partial}{\partial s}\langle\beta f\rangle \frac{\partial}{\partial s}+\gamma\right)\langle u\rangle \text { on } \Gamma_{0}
\end{aligned}
$$

where $\alpha=2 \delta\left\langle f\left(\mu_{0}-\mu\right)\right\rangle, \beta^{ \pm}=2 \delta\left(\frac{1}{\mu_{0}}-\frac{1}{\mu^{ \pm}}\right)$and $\gamma=2 \delta k^{2}\left\langle f\left(n-n_{0}\right)\right\rangle$.

It is worthwhile noticing that all the three coefficients involved in the expression of the ATCs depend on the thickness and the shape of the defect $\Omega_{0}$, as well as on the contrasts between material properties of the two delaminated layers $\Omega_{ \pm}$and the original thin delamination $\Omega_{0}$.

REMARK 2.1. We remark that our asymptotic expressions along with the derivation of the ATCs are merely formal. Although not needed to write down the final asymptotic model, in our derivation process we have used that the functions $f^{ \pm}$are regular at the end points of $\Gamma_{0}$ meaning in particular that $f^{ \pm}(0)=f^{ \pm}(L)=0$. In the case of regular $f^{ \pm}$, a rigorous justification of the asymptotic model can be done following the approach in [21, 20] for periodic interfaces with constant width.

2.2. Formulation of the approximate model. We can now replace the original problem (1.1)-(1.4), (1.5)-(1.8) and (1.9) by an approximate problem, here referred to as the crack problem, where the opening $\Omega_{0}$ is replaced by the portion $\Gamma_{0}$ of $\Gamma$ where the fields satisfy the jump conditions derived above. In an abuse of notation, from now on $u^{ \pm}$will refer to the solution of the approximate problem. We define then the forward approximate scattering problem (i.e. the crack problem), that reads: given the plane wave incident field $u^{i}(x):=e^{i k x \cdot d}$ find the total fields $u^{e x t}=u^{s}+u^{i}, u^{+}$ and $u^{-}$satisfying

$$
\begin{aligned}
& \Delta u^{e x t}+k^{2} u^{e x t}=0 \quad \text { in } \Omega_{\mathrm{ext}} \\
& \nabla \cdot\left(\frac{1}{\mu_{+}} \nabla u^{+}\right)+k^{2} n_{+} u^{+}=0 \quad \text { in } \quad \Omega_{+} \\
& \nabla \cdot\left(\frac{1}{\mu_{-}} \nabla u^{-}\right)+k^{2} n_{-} u^{-}=0 \quad \text { in } \quad \Omega_{-}
\end{aligned}
$$


and

$$
\begin{array}{cc}
u^{e x t}=u^{+} \quad \text { and } \frac{\partial u^{e x t}}{\partial \nu}=\frac{1}{\mu_{+}} \frac{\partial u^{+}}{\partial \nu} & \text { on } \Gamma_{1} \\
{[u]=0 \quad \text { and } \quad\left[\frac{1}{\mu} \frac{\partial u}{\partial \nu}\right]=0} & \text { on } \Gamma \backslash \bar{\Gamma}_{0} \\
{[u]=\alpha\left\langle\frac{1}{\mu} \frac{\partial u}{\partial \nu}\right\rangle \quad \text { and } \quad\left[\frac{1}{\mu} \frac{\partial u}{\partial \nu}\right]=\left(-\frac{\partial}{\partial s}\langle\beta f\rangle \frac{\partial}{\partial s}+\gamma\right)\langle u\rangle} & \text { on } \Gamma_{0}
\end{array}
$$

along with the Sommerfeld radiation condition (1.9) for the scattered field $u^{s}$ (see Figure 3), where we recall $[w]=w^{+}-w-$ and $\langle w\rangle=\left(w^{+}+w^{-}\right) / 2$, and

$$
\alpha=2 \delta\left\langle f\left(\mu_{0}-\mu\right)\right\rangle, \quad \beta^{ \pm}=2 \delta\left(\frac{1}{\mu_{0}}-\frac{1}{\mu^{ \pm}}\right), \quad \gamma=2 \delta k^{2}\left\langle f\left(n-n_{0}\right)\right\rangle .
$$

We remark that although our formal asymptotic calculations are performed only in

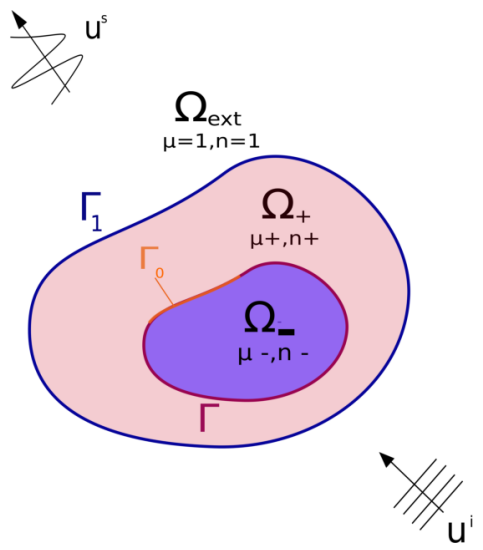

FIG. 3. The configuration of the crack problem.

the two-dimensional case, for the analysis in the following will assume that the approximate model (2.10)-(2.12), (2.13)-(2.15) and (1.9) is valid in the three-dimensional case also. Of course in the three-dimensional case the boundary differential operator $\partial / \partial s\langle\beta f\rangle \partial / \partial s$ is replaced by the Laplace-Beltrami operator in the divergence form $\nabla_{\Gamma} \cdot\langle\beta f\rangle \nabla_{\Gamma}$, i.e. $(2.15)$ is replaced by

$$
[u]=\alpha\left\langle\frac{1}{\mu} \frac{\partial u}{\partial \nu}\right\rangle \quad \text { and } \quad\left[\frac{1}{\mu} \frac{\partial u}{\partial \nu}\right]=\left(-\nabla_{\Gamma} \cdot\langle\beta f\rangle \nabla_{\Gamma}+\gamma\right)\langle u\rangle \quad \text { on } \Gamma_{0}
$$

where $\nabla_{\Gamma}$ and $\nabla_{\Gamma}$ are the surface divergence and the surface gradient on $\Gamma$, respectively.

2.3. Numerical validation of the approximate model. We end this section with a numerical study of the convergence of the approximate crack problem to the original problem as $\delta \rightarrow 0$ in the two-dimensional case. Again ignoring the effect of the end points of $\Gamma_{0}$ on the asymptotic expansions, heuristically it is expected that the order of convergence is $\delta^{2}$. To validate the ATCs, we compare the solution of the scattering problem by a finite element method based on directly meshing the opening $\Omega_{0}$ (i.e. solving (1.1)-(1.4), (1.5)-(1.8) and (1.9) by a finite element method) 
to the solution of the crack problem (i.e. (2.10)-(2.12), (2.13)-(2.15) and (1.9) by a finite element based on the variational problem (3.10)). Both problems are solved using a FEM code where the unbounded domain is truncated and the exact boundary condition in terms of Dirichlet-to-Neumann operator (which is explained in more detail in the following section) is imposed on a circular artificial boundary.

For our numerical example we consider a circular inhomogeneity of radius one with an opening $\Omega_{0}$ given by (see Figure 2.3 )

$$
f^{-}(s)=0, f^{+}(s):=-l^{-2}(s+l)(s-l) ; \text { for } s \in(-l, l), \text { with } l=0.2 \pi,
$$

on the interface $r=1$. The material properties are chosen to be $n_{-}=1, \mu_{-}=1$ in $\Omega_{-}, n_{+}=1, \mu^{+}=1$ in $\Omega_{+}, n_{0}=0.2, \mu_{0}=0.9$ in $\Omega_{0}$, and the wave number $k=3$. For

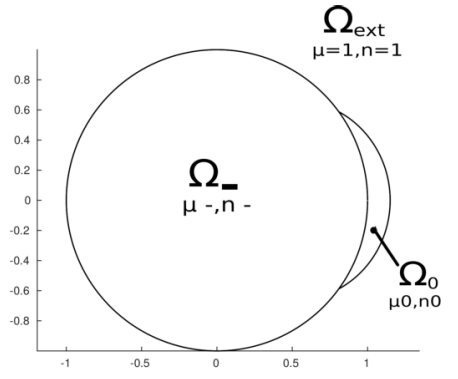

FIG. 4. The configuration of the delaminated structure used in the numerical experiments

fixed $\delta=0.04 \lambda$ (where $\lambda=2 \pi / k$ is the wave length) and different incident directions $d=(\cos (\theta), \sin (\theta))$, in Figure 5 panel (a) we plot the $H^{1}$ relative error

$$
e(d, \delta):=\frac{\left\|u_{\delta}^{e x t}-u^{e x t}\right\|_{H^{1}\left(B_{R} \backslash \bar{\Omega}\right)}}{\left\|u^{e x t}\right\|_{H^{1}\left(B_{R} \backslash \bar{\Omega}\right)}}
$$

where $u_{\delta}^{e x t}$ and $u^{\text {ext }}$ correspond to the exact scattering problem (1.1)-(1.4), (1.5)(1.8), (1.9)) and to the approximate scattering problem (2.10)-(2.12), (2.13)-(2.15), (1.9)), respectively, and $B_{R}$ is a large ball of radius $R>0$ containing $\Omega=\Omega_{+} \cup \Omega_{-}$. We observe that the maximum error is attained for the incident direction $d=(1,0)$, i.e. for the incident plane wave $u^{i}(x, y)=e^{i k x}$ which hits the opening $\Omega_{0}$ in the middle in perpendicular direction. Figure 5 panel (b) shows the $H^{1}$ relative error $e(d, \delta)$ as a function of the small parameter $\delta$ corresponding to the incident direction $d=(1,0)$. The plot shows that the numerical convergence rate is close to $O\left(\delta^{1.7}\right)$ which approximately corresponds to the expected theoretical rate of convergence rate $O\left(\delta^{2}\right)$ for the second order ATCs model. Since for the solution of inverse problem we use far field data, which is defined in Section 4, in Figure 6 we show numerical results where we compare the far fields of the exact model and the approximate model for the same shape as above. In Figure 6 panel (a) it is shown the absolute value of the far fields $u_{\delta}^{\infty}(\cdot, d)$ and $u^{\infty}(\cdot, d)$ corresponding to the scattered waves for the ATCs model and the exact model, respectively, again for $d=(1,0)$. In Figure 6 panel (b) we show the relative $L^{2}$ error of these far fields

$$
e^{\infty}(\delta, d):=\frac{\left\|u_{\delta}^{\infty}-u^{\infty}\right\|_{L^{2}\left(\mathbb{S}^{1}\right)}}{\left\|u^{\infty}\right\|_{L^{2}\left(\mathbb{S}^{1}\right)}}
$$

for different values of $\delta$ and $d=(1,0)$. The plot shows that the numerical convergence rate of the far fields in approximately $O\left(\delta^{1}\right)$. 


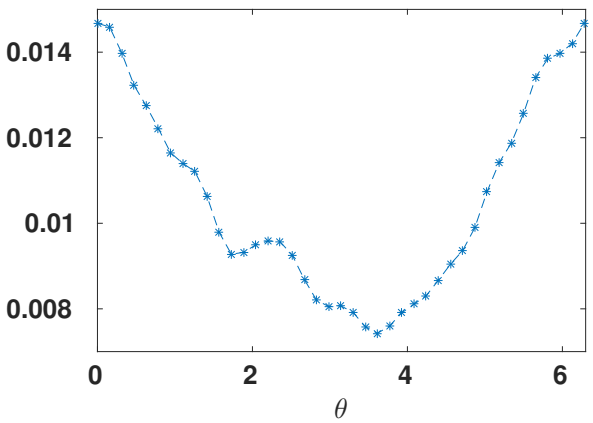

(a)

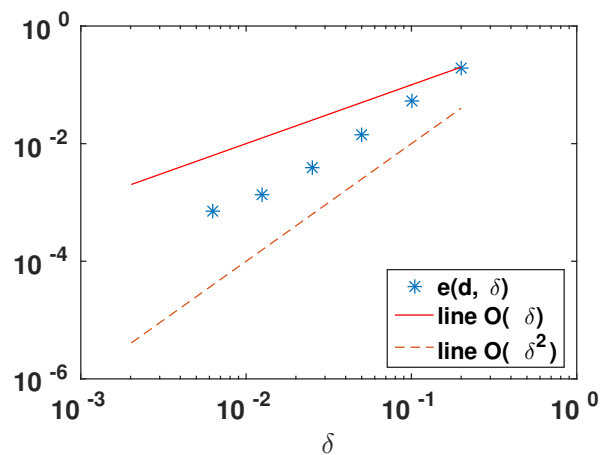

(b)

Fig. 5. Panel (a) shows the $H^{1}$ relative error of total fields resulting from different incident direction, whereas panel $(b)$ the $H^{1}$ relative error for different values of $\delta$. The approximated rate of convergence is $O\left(\delta^{1.7}\right)$.

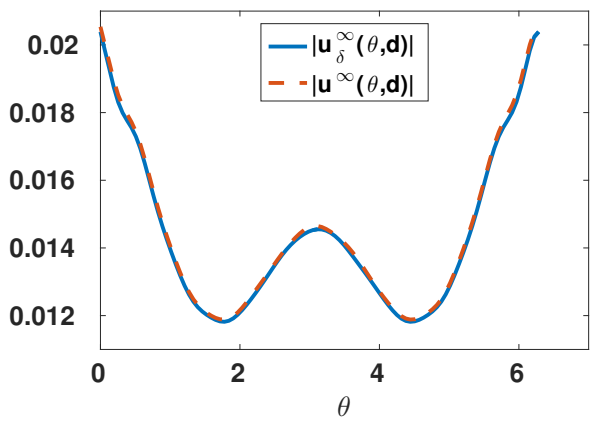

(a)

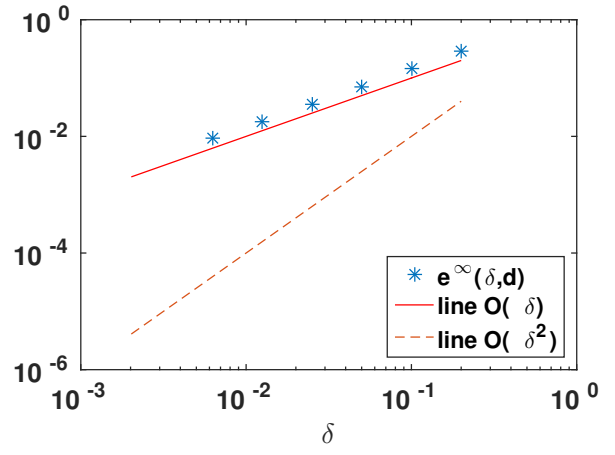

(b)

FIG. 6. Panel (a) shows the plot of the absolute value of the far field for both models for $\delta=0.05$. Panel (b) shows the far field $L^{2}$ relative error $e^{\infty}(\delta, d)$, for different values of $\delta$. The approximated rate of convergence is $O\left(\delta^{1}\right)$.

3. The well-posedness of the approximate model. Now we turn our attention to the study of the well-posedness of the approximate crack problem (2.10)-(2.12), (2.13)-(2.15) and (1.9). Although our formal asymptotic calculations are preformed only in the two-dimensional case, for the analysis we shall assume that this approximate model is also valid in the three-dimensional case. To study the problem we employ a variational method which provides also the analytical framework for a finite element method to numerically compute the solution. The first step is to formulate the problem in a bounded domain and to this end we introduce a large ball $B_{R}$ of radius $R>0$ containing $\bar{\Omega}$ and let $S_{R}$ denote the boundary of $B_{R}$. The exterior Dirichlet-to-Neumann operator $T_{k}: H^{1 / 2}\left(S_{R}\right) \rightarrow H^{-1 / 2}\left(S_{R}\right)$ is defined by

$$
T_{k}: \alpha \mapsto \frac{\partial v}{\partial \nu} \quad \text { on } \quad S_{R}
$$


where $v \in H_{l o c}^{1}\left(\mathbb{R}^{m} \backslash \bar{B}_{R}\right)$ solves

$$
\begin{aligned}
\Delta v+k^{2} v=0 & \text { in } \quad \mathbb{R}^{m} \backslash \bar{B}_{R} \\
v=\alpha & \text { on } \quad S_{R} \\
\lim _{r \rightarrow \infty} r^{\frac{m-1}{2}}\left(\frac{\partial u^{s}}{\partial r}-i k u^{s}\right)=0 . &
\end{aligned}
$$

It is well-known that the exterior Dirichlet-to-Neumann operator $T_{k}: H^{1 / 2}\left(S_{R}\right) \rightarrow$ $H^{-1 / 2}\left(S_{R}\right)$ satisfies (see e.g [14])

$$
\Im\left(\int_{S_{R}}\left(T_{k} u\right) \bar{u} d s\right) \geq 0 \quad \text { and } \quad-\Re\left(\int_{S_{R}}\left(T_{k} u\right) \bar{u} d s\right) \geq 0 .
$$

It is standard to show (see e.g. [13] and [14]) that (2.10)-(2.12), (2.13)-(2.15) and (1.9) is equivalent to the problem of finding $u^{\text {ext }}, u^{+}, u^{-}$satisfying

$$
\begin{aligned}
& \Delta u^{e x t}+k^{2} u^{e x t}=0 \quad \text { in } \quad B_{R} \backslash \bar{\Omega} \\
& \nabla \cdot\left(\frac{1}{\mu_{+}} \nabla u^{+}\right)+k^{2} n_{+} u^{+}=0 \quad \text { in } \quad \Omega_{+} \\
& \nabla \cdot\left(\frac{1}{\mu_{-}} \nabla u^{-}\right)+k^{2} n_{-} u^{-}=0 \quad \text { in } \quad \Omega_{-} \\
& \frac{\partial\left(u^{e x t}-u_{i}\right)}{\partial \nu}=T_{k}\left(u^{e x t}-u^{i}\right) \quad \text { on } \quad S_{R} \\
& u^{e x t}=u^{+} \quad \text { and } \quad \frac{\partial u^{e x t}}{\partial \nu}=\frac{1}{\mu_{+}} \frac{\partial u^{+}}{\partial \nu} \quad \text { on } \quad \Gamma_{1} \\
& {[u]=0 \quad \text { and } \quad\left[\frac{1}{\mu} \frac{\partial u}{\partial \nu}\right]=0 \quad \text { on } \quad \Gamma \backslash \bar{\Gamma}_{0}} \\
& {[u]=\alpha\left\langle\frac{1}{\mu} \frac{\partial u}{\partial \nu}\right\rangle \quad \text { and } \quad\left[\frac{1}{\mu} \frac{\partial u}{\partial \nu}\right]=\left(-\nabla_{\Gamma} \cdot\langle\beta f\rangle \nabla_{\Gamma}+\gamma\right)\langle u\rangle \quad \text { on } \quad \Gamma_{0} .}
\end{aligned}
$$

In $\mathbb{R}^{2}$ the boundary differential operator simplifies to

$$
\nabla_{\Gamma} \cdot\langle\beta f\rangle \nabla_{\Gamma} w=\frac{\partial}{\partial s}\langle\beta f\rangle \frac{\partial}{\partial s} w .
$$

We recall that $\Omega=\Omega_{+} \cup \Omega_{-}$and the coefficients $\alpha,\langle\beta f\rangle$ and $\gamma$, which are bounded functions defined on $\Gamma_{0}$, are given by (2.16). In order to study the well-posedeness of the above problem, we notice that while the energy space $H^{1}$ suffices to rigorously define the solution of the differential equations in $\Omega_{ \pm}$and $B_{R} \backslash \bar{\Omega}$, it is not enough to define the boundary differential operator on $\Gamma_{0}$ that appears in (3.8). To handle the boundary differential operator on $\Gamma_{0}$ we define the space

$$
\mathcal{H}:=\left\{u \in H^{1}\left(B_{R} \backslash \bar{\Gamma}_{0}\right) \text { such that } \sqrt{f^{ \pm}} \nabla_{\Gamma}\langle u\rangle \in L^{2}\left(\Gamma_{0}\right)\right\}
$$

endowed with the norm

$$
\|u\|_{\mathcal{H}}^{2}=\|u\|_{H^{1}\left(B_{R} \backslash \bar{\Gamma}_{0}\right)}^{2}+\left\|\sqrt{f^{+}} \nabla_{\Gamma}\langle u\rangle\right\|_{L^{2}\left(\Gamma_{0}\right)}^{2}+\left\|\sqrt{f^{-}} \nabla_{\Gamma}\langle u\rangle\right\|_{L^{2}\left(\Gamma_{0}\right)}^{2} .
$$


Obviously $\mathcal{H}$ is a Hilbert space since the weights $f^{ \pm} \in L^{\infty}\left(\Gamma_{0}\right)$ are non-negative (note that $f^{ \pm}=0$ at the boundary of $\Gamma_{0}$ on $\Gamma$ ). Now, multiplying all three equations with $v \in \mathcal{H}$, integrating by parts, using the continuity of transmission conditions across $\Gamma \backslash \bar{\Gamma}_{0}$, the boundary condition on $S_{R}$, and the approximate transmission condition on $\Gamma_{0}$, we arrive at the following equivalent variational formulation of (3.2)-(3.8): find $u \in \mathcal{H}$ such that

$$
A(u, v)=L(v) \quad \text { for all } v \in \mathcal{H}
$$

where

$$
\begin{aligned}
A(u, v) & :=\int_{B_{R}} \frac{1}{\mu} \nabla u \cdot \overline{\nabla v}-k^{2} n u \bar{v} \mathrm{~d} x+\int_{\Gamma_{0}}\langle\beta f\rangle \nabla_{\Gamma}\langle u\rangle \nabla_{\Gamma} \overline{\langle v\rangle} d s \\
& +\int_{\Gamma_{0}} \gamma\langle u\rangle \overline{\langle v\rangle} \mathrm{d} s+\int_{\Gamma_{0}} \frac{1}{\alpha}[u] \overline{[v]} \mathrm{d} s-\int_{S_{R}} T_{k} u \bar{v} \mathrm{~d} s
\end{aligned}
$$

and

$$
L(v)=-\int_{S_{R}}\left(T_{k} u^{i} \bar{v}-\frac{\partial u^{i}}{\partial \nu} \bar{v}\right) d s .
$$

Here $\left.u\right|_{\Omega_{+}}=u^{+},\left.u\right|_{\Omega_{-}}=u^{-}$and $\left.u\right|_{B_{R} \backslash \bar{\Omega}}=u^{e x t}$, and

$$
\mu:=1, n:=1 \text { in } B_{R} \backslash \bar{\Omega}, \mu:=\mu_{+}, n:=n_{+} \text {in } \Omega_{+}, \mu:=\mu_{-}, n:=n_{-} \text {in } \Omega_{-} .
$$

We decompose the bounded sesquilinear form $A: \mathcal{H} \times \mathcal{H} \rightarrow \mathbb{C}$ defined by (3.17) as

$$
A(u, v)=A_{0}(u, v)+B(u, v),
$$

where

$$
A_{0}(u, v):=\int_{B_{R}} \frac{1}{\mu} \nabla u \cdot \overline{\nabla v}+u \bar{v} \mathrm{~d} x+\int_{\Gamma_{0}}\langle\beta f\rangle \nabla_{\Gamma}\langle u\rangle \nabla_{\Gamma} \overline{\langle v\rangle} \mathrm{d} s-\int_{S_{R}} T_{k} u \bar{v} d s
$$

and

$$
B(u, v):=-\int_{B_{R}}\left(k^{2} n+1\right) u \bar{v} \mathrm{~d} x+\int_{\Gamma_{0}} \gamma\langle u\rangle \overline{\langle v\rangle} \mathrm{d} s+\int_{\Gamma_{0}} \frac{1}{\alpha}[u] \overline{[v]} \mathrm{d} s .
$$

Let $\mathbb{A}_{0}: \mathcal{H} \rightarrow \mathcal{H}$ and $\mathbb{B}: \mathcal{H} \rightarrow \mathcal{H}$ be the linear operators defined from the sesquilinear forms $A_{0}(\cdot, \cdot)$ and $B(\cdot, \cdot)$ by means of the Riesz representation theorem

$$
\left(\mathbb{A}_{0} u, v\right)_{\mathcal{H}}=A_{0}(u, v) \quad \text { and } \quad(\mathbb{B} u, v)_{\mathcal{H}}=B(u, v), \quad \text { for all } u, v \in \mathcal{H} .
$$

At this point let us assume that there exist constants $\epsilon_{1}>0$ and $\epsilon_{2}>0$ such that $\Re\left(\frac{1}{\mu}\right) \geq \epsilon_{1}$, and $\Re\left(\frac{1}{\mu_{0}}-\frac{1}{\mu^{ \pm}}\right) \geq \epsilon_{2}$ (which implies that $\Re\left(\beta^{ \pm}\right) \geq 2 \delta \epsilon_{2}$ ). Then we have that

$$
\begin{aligned}
\Re\left(A_{0}(u, u)\right) & =\int_{B_{R}}\left(\Re\left(\frac{1}{\mu}\right)|\nabla u|^{2}+|u|^{2}\right) \mathrm{d} x+\int_{\Gamma_{0}}\langle\Re(\beta) f\rangle\left|\nabla_{\Gamma}\langle u\rangle\right|^{2} d s \\
& -\Re\left(\int_{S_{R}}\left(T_{k} u\right) \bar{u} \mathrm{~d} s\right) \\
& \geq \min \left(\epsilon_{1}, 1\right)\|u\|_{H^{1}(\Omega)}^{2}+\delta \epsilon_{2}\left\|\sqrt{f^{+}} \nabla_{\Gamma}\langle u\rangle\right\|_{L^{2}\left(\Gamma_{0}\right)}^{2} \\
& +\delta \epsilon_{2}\left\|\sqrt{f^{-}} \nabla_{\Gamma}\langle u\rangle\right\|_{L^{2}\left(\Gamma_{0}\right)}^{2} \geq C\|u\|_{\mathcal{H}}^{2}
\end{aligned}
$$


for some positive constant $C>0$, which proves that $A_{0}(\cdot, \cdot)$ is coercive. The boundedness of $A_{0}(\cdot, \cdot)$ is obvious given the assumptions on the coefficients and the fact that $T_{k}$ is bounded. Thus, $\mathbb{A}_{0}: \mathcal{H} \rightarrow \mathcal{H}$ is invertible operator with bounded inverse.

Due to the fact that $\alpha:=2 \delta\left\langle f\left(\mu_{0}-\mu\right)\right\rangle$ is zero at the boundary of $\Gamma_{0}$ in $\Gamma$, the operator $\mathbb{B}$ is not bounded in general. We need to impose some restriction on the rate that $f^{ \pm}$approaches zero at boundary of $\Gamma_{0}$. Indeed we can prove the following result:

Lemma 3.1. Assume $1 / \alpha \in L^{t}\left(\Gamma_{0}\right)$ for $t=1+\epsilon$ in $\mathbb{R}^{2}$ and $t=7 / 4+\epsilon$ in $\mathbb{R}^{3}$ for arbitrary small $\epsilon>0$. Then $\mathbb{B}: \mathcal{H} \rightarrow \mathcal{H}$ is a compact bounded linear operator.

Proof. We check all three terms of the operator $\mathbb{B}$, i.e.

$$
\begin{gathered}
\left(\mathbb{B}_{1} u, v\right)_{\mathcal{H}}=-\int_{B_{R}}\left(k^{2} n+1\right) u \bar{v} \mathrm{~d} x, \quad\left(\mathbb{B}_{2} u, v\right)_{\mathcal{H}}=\int_{\Gamma_{0}} \gamma\langle u\rangle \overline{\langle v\rangle} \mathrm{d} s \\
\text { and } \quad\left(\mathbb{B}_{3} u, v\right)_{\mathcal{H}}=\int_{\Gamma_{0}} \frac{1}{\alpha}[u] \overline{[v]} \mathrm{d} s .
\end{gathered}
$$

Noting that $n \in L^{\infty}\left(B_{R}\right)$, the compactness of $\mathbb{B}_{1}$ follows from the fact that $H^{1}\left(B_{R}\right)$ (and consequently $\mathcal{H}$ ) is compactly embedded in $L^{2}\left(B_{R}\right)$ and that

$$
\left\|\mathbb{B}_{1} u\right\|_{\mathcal{H}}=\sup _{\|v\|_{\mathcal{H}}=1}\left|-\int_{B_{R}}\left(k^{2} n+1\right) u \bar{v} d x\right| \leq C\|u\|_{L^{2}\left(B_{R}\right)} .
$$

Next, since $\gamma \in L^{\infty}\left(\Gamma_{0}\right)$, we have that

$$
\begin{aligned}
\left\|\mathbb{B}_{2} u\right\|_{\mathcal{H}} & =\sup _{\|v\|_{\mathcal{H}}=1}\left|\int_{\Gamma_{0}} \gamma\langle u\rangle \overline{\langle v\rangle} \mathrm{d} s\right| \leq C \sup _{\|v\|_{\mathcal{H}}=1}\|\langle u\rangle\|_{L^{2}\left(\Gamma_{0}\right)}\|\langle v\rangle\|_{L^{2}\left(\Gamma_{0}\right)} \\
& \leq C \sup _{\|v\|_{\mathcal{H}}=1}\|\langle u\rangle\|_{L^{2}(\Gamma)}\|\langle v\rangle\|_{H^{1 / 2}(\Gamma)} \\
& \leq C \sup _{\|v\|_{\mathcal{H}}=1}\|\langle u\rangle\|_{L^{2}(\Gamma)}\|v\|_{H^{1}\left(B_{R}\right)} \leq C\|\langle u\rangle\|_{L^{2}(\Gamma)} .
\end{aligned}
$$

for some positive constant $C>0$, where we have used the continuity of the trace operator from $H^{1}\left(B_{R}\right)$ to $H^{1 / 2}(\Gamma)$. Now the compactness of $\mathbb{B}_{2}$ follows from the the boundedness of the trace operator and compactly embedding of $H^{1 / 2}(\Gamma)$ into $L^{2}(\Gamma)$.

Due to the fact that $\alpha:=2 \delta\left\langle f\left(\mu_{0}-\mu\right)\right\rangle$ is zero at the boundary of $\Gamma_{0}$ in $\Gamma$, the analysis of $\mathbb{B}_{3}$ is more delicate and we need to appeal to Rellich-Kondrachov embedding theorems for $W^{m, p}$ spaces (see e.g. [1]). To this end we first recall that from Theorem 5.3 of [1], we have that for a bounded domain $\mathcal{O}$ with $C^{1}$-boundary $\partial \mathcal{O}$, the trace operator $\gamma: H^{1}(\mathcal{O}) \rightarrow L^{q}(\partial \mathcal{O})$ is a continuous embedding if $2 \leq q<\infty$ for $\mathcal{O} \subset \mathbb{R}^{2}$, and $2 \leq q<4$ for $\mathcal{O} \subset \mathbb{R}^{3}$. Hence assuming that $\Gamma_{0}$ is smooth and using this embedding result, for $t$ as in the assumptions of the lemma we have that

$$
\begin{aligned}
\left\|\mathbb{B}_{3} u\right\|_{\mathcal{H}} & =\sup _{\|v\|_{\mathcal{H}}=1}\left|\int_{\Gamma_{0}} \frac{1}{\alpha}[u][\bar{v}] d s\right| \leq \sup _{\|v\|_{\mathcal{H}}=1}\left\|\frac{1}{\alpha}\right\|_{L^{t}\left(\Gamma_{0}\right)}\|[v]\|_{L^{p}\left(\Gamma_{0}\right)}\|[u]\|_{L^{q}\left(\Gamma_{0}\right)} \\
& \leq C \sup _{\|v\|_{\mathcal{H}}=1}\left\|\frac{1}{\alpha}\right\|_{L^{t}\left(\Gamma_{0}\right)}\|v\|_{\mathcal{H}}\|[u]\|_{L^{q}\left(\Gamma_{0}\right)} \leq C\left\|\frac{1}{\alpha}\right\|_{L^{t}\left(\Gamma_{0}\right)}\|[u]\|_{L^{q}\left(\Gamma_{0}\right)} .
\end{aligned}
$$

where we have used that there is a constant $C>0$, such that $\|[v]\|_{L^{p}\left(\Gamma_{0}\right)} \leq C\|v\|_{\mathcal{H}}$. Note that for arbitrary small $\epsilon, p$ and $q$ are chosen arbitrarily large in $\mathbb{R}^{2}$ and arbitrarily close to 4 in $\mathbb{R}^{3}$, in both cases such that $1 / t+1 / p+1 / q=1$. We also remark 
that for $u \in \mathcal{H}$ we have that $[u]=0$ in $\Gamma \backslash \Gamma_{0}$. Now, we use the Rellich-Kondrachov compact embedding theorem (see Theorem 6.3, Part I in [1]). Applying this theorem for $\Omega=\Omega_{0}:=\Gamma_{0}$ which is a 2-d smooth manifold in the case of $\mathbb{R}^{3}$ or 1 -d smooth manifold in the case of $\mathbb{R}^{2}$ (in our case $m=1 / 2, p=2, j=0, k=n=2$ in $\mathbb{R}^{3}$ or $k=n=1$ in $\mathbb{R}^{2}$ ), implies that the embedding

$$
H^{1 / 2}\left(\Gamma_{0}\right) \hookrightarrow L^{q}\left(\Gamma_{0}\right)
$$

is compact if $1 \leq q<4$ in $\mathbb{R}^{3}$ or if $1 \leq q<\infty$ in $\mathbb{R}^{2}$. Combining this fact with the fact that embedding $\mathcal{H} \hookrightarrow H^{1 / 2}\left(\Gamma_{0}\right)$ is bounded in (3.16) proves that $\mathbb{B}_{3}$ is compact, and this concludes the proof of the lemma. We remark that here Theorem 6.3, [1] is adapted to the compact manifold $\Gamma_{0}$ covered by a finite number of charts each with Riemannian metric bounded below and above by the Euclidean metric, by applying standard arguments based on the partition of unity. $\square$

Lemma 3.2. Assume that $0 \leq \Im\left(n^{ \pm}\right) \leq \Im\left(n_{0}\right)$ and $0 \leq \Im\left(\mu^{ \pm}\right) \leq \Im\left(\mu_{0}\right)$. Then the problem (3.2)-(3.8) has a unique solution.

Proof. Take $u^{i}=0$ in (3.2)-(3.8), and let $u$ be a solution to the homogenous problem. Taking the imaginary part of (3.17) for $v=u$ we have

$$
\begin{aligned}
0 & =\int_{B_{R}} \Im\left(\frac{1}{\mu}\right)|\nabla u|-k^{2} \Im(n)|u|^{2} \mathrm{~d} x+\int_{\Gamma_{0}} \Im\langle\beta f\rangle\left|\nabla_{\Gamma}\langle u\rangle\right|^{2} d s \\
& +\int_{\Gamma_{0}} \Im(\gamma)|\langle u\rangle|^{2} \mathrm{~d} s+\int_{\Gamma_{0}} \Im\left(\frac{1}{\alpha}\right)|[u]|^{2} \mathrm{~d} s-\Im\left(\int_{S_{R}} T_{k} u \bar{u} \mathrm{~d} s\right)
\end{aligned}
$$

Now, since from the assumptions on the material properties we have that $\Im\left(\frac{1}{\mu^{ \pm}}\right) \leq 0$, $\Im\left(n^{ \pm}\right) \geq 0, \Im(\langle\beta f\rangle) \leq 0, \Im(\alpha) \geq 0$ and $\Im(\gamma) \leq 0$, the above equation implies

$$
\Im\left(\int_{S_{R}} T_{k} u \bar{u} \mathrm{~d} s\right) \leq 0 .
$$

But (3.1) now implies that indeed

$$
\Im\left(\int_{S_{R}} T_{k} u \bar{u} \mathrm{~d} s\right)=0 .
$$

The definition of the Dirichet-to-Neumann operator and Rellich's Lemma (see [13] and [18]) now imply that $u=0$ and $\partial u / \partial \nu=0$ on $S_{R}$. Finally, from Holmgren's theorem together with the unique continuation principle (which under our geometrical and physical assumptions holds true, see e.g. Theorem 17.2.6 in [26]), we can conclude that $u=0$ which proves the uniqueness of (3.2)-(3.8).

In summary, combining Lemma 3.1 and Lemma 3.2 with the coercivity result (3.15) we obtain the main result of this section.

TheOREm 3.3 (Well-posedness). In addition to the geometrical and physical assumptions stated in the Introduction, assume that:

1. $\Re\left(\frac{1}{\mu}\right) \geq \epsilon_{1}$, and $\Re\left(\frac{1}{\mu_{0}}-\frac{1}{\mu^{ \pm}}\right) \geq \epsilon_{2}$ for some constants $\epsilon_{1}>0$ and $\epsilon_{2}>0$,

2. $0 \leq \Im\left(n^{ \pm}\right) \leq \Im\left(n_{0}\right)$ and $0 \leq \Im\left(\mu^{ \pm}\right) \leq \Im\left(\mu_{0}\right)$ and

3. the profile $f^{ \pm}$go to zero at the boundary of $\Gamma_{0}$ in $\Gamma$ such that $1 / \alpha \in L^{t}\left(\Gamma_{0}\right)$ for $t=1+\epsilon$ in $\mathbb{R}^{2}$ and $t=7 / 4+\epsilon$ in $\mathbb{R}^{3}$ for arbitrary small $\epsilon>0$, where $\alpha=\left\langle f\left(\mu_{0}-\mu\right)\right\rangle$. 
Then the problem (3.2)-(3.8) has a unique solution $u \in \mathcal{H}$ which depends continuously on the incident wave $u^{i}$ with respect to the $\mathcal{H}$-norm.

REMARK 3.1. Since any solution of (3.2)-(3.8) can be extended to a solution of the scattering problem (2.10)-(2.12), (2.13)-(2.15) and (1.9) and vise-versa, Theorem (3.3) provides a well-posedness result for the approximate crack problem.

For later use we need to consider the above scattering problem in the following form: Find $w \in \mathcal{H} \cap H_{\text {loc }}^{1}\left(\mathbb{R}^{m} \backslash \Gamma_{0}\right)$ such that

$$
\begin{gathered}
\nabla \cdot\left(\frac{1}{\mu} \nabla w\right)+k^{2} n w=0 \quad \text { in } \quad \mathbb{R}^{m} \backslash \bar{\Gamma}_{0}, \\
{[w]=\alpha\left\langle\frac{1}{\mu} \frac{\partial w}{\partial \nu}\right\rangle+\alpha h_{1} \quad \text { on } \quad \Gamma_{0},} \\
{\left[\frac{1}{\mu} \frac{\partial w}{\partial \nu}\right]=\left(-\nabla_{\Gamma} \cdot\langle\beta f\rangle \nabla_{\Gamma}+\gamma\right)\langle w\rangle+h_{2} \quad \text { on } \quad \Gamma_{0},} \\
\lim _{r \rightarrow \infty} r^{\frac{m-1}{2}}\left(\frac{\partial w}{\partial r}-i k w\right)=0,
\end{gathered}
$$

where $h_{1}$ and $h_{2}$ are

$$
\left\{\begin{array}{c}
h_{1}:=\left\langle\frac{1}{\mu} \frac{\partial v}{\partial \nu}\right\rangle-\frac{1}{\alpha}[v] \\
h_{2}:=\left(-\nabla_{\Gamma} \cdot\langle\beta f\rangle \nabla_{\Gamma}+\gamma\right)\langle v\rangle-\left[\frac{1}{\mu} \frac{\partial v}{\partial \nu}\right]
\end{array}\right.
$$

for some $v \in \mathcal{H}$ with $\nabla \cdot((1 / \mu) \nabla v) \in L^{2}\left(B_{R} \backslash \Gamma_{0}\right)$. For the later use we define the following trace space on $\Gamma_{0}$ of function $u \in \mathcal{H}$,

$$
\mathcal{H}\left(\Gamma_{0}\right):=\left\{u \in H^{1 / 2}\left(\Gamma_{0}\right) \text { such that } \sqrt{f^{ \pm}} \nabla_{\Gamma} u \in L^{2}\left(\Gamma_{0}\right)\right\}
$$

and its dual $\mathcal{H}^{-1}\left(\Gamma_{0}\right)$ with respect to the following duality pairing

$$
(u, v)_{\mathcal{H}\left(\Gamma_{0}\right), \mathcal{H}^{-1}\left(\Gamma_{0}\right)}:=(u, v)_{H^{1 / 2}\left(\Gamma_{0}\right), \tilde{H}^{-1 / 2}\left(\Gamma_{0}\right)}+\left(f^{ \pm} \nabla_{\Gamma} u, \nabla_{\Gamma} v\right)_{L^{2}\left(\Gamma_{0}\right), L^{2}\left(\Gamma_{0}\right)} .
$$

Here $\tilde{H}^{1 / 2}\left(\Gamma_{0}\right)$ and $\tilde{H}^{-1 / 2}\left(\Gamma_{0}\right)$ consist of functions in $H^{1 / 2}\left(\Gamma_{0}\right)$ and $H^{-1 / 2}\left(\Gamma_{0}\right)$ that can be extended by zero in the entire $\Gamma$ as $H^{1 / 2}$ and $H^{-1 / 2}$ functions, respectively. They are duals of $H^{-1 / 2}\left(\Gamma_{0}\right)$ and $H^{1 / 2}\left(\Gamma_{0}\right)$, respectively. Hence $h_{1} \in H^{-1 / 2}\left(\Gamma_{0}\right)$ and $h_{2} \in \mathcal{H}^{-1}\left(\Gamma_{0}\right)$.

4. The inverse problem of reconstructing the delaminated part $\Gamma_{0}$. In this section we turn our attention to the main goal of this study, which is the reconstruction of the delaminated portion $\Gamma_{0}$ of the interface $\Gamma$ between two materials from measured scattering data. Our reconstruction method is a modified linear sampling method, adapted to our problem where we already know the interface $\Gamma$ and only look for the delaminated part $\Gamma_{0}$. The linear sampling method and factorization method have been used to reconstruct cracks or screens with various types of boundary conditions [8], [10], [12], [27] and [33] (see also the monographs [13] and [15]). Although numerically both the linear sampling method and factorization method provide similar reconstruction results, the factorization method is mathematically more satisfactory. Here we develop the linear sampling method since our complicated jump conditions 
modeling the delaminated part $\Gamma_{0}$ fail to satisfy the standard assumptions under which the factorization method works (see [17]). For other inversion methods applied to similar types of inverse problems in acoustic and elasticity we refer the reader to $[2,3,6]$.

We assume that the interrogating incident fields are plane waves given by $u^{i}(x, d)=$ $e^{i k d \cdot x}$ where the unit vector $d$ is the incident direction. The corresponding scattered field $u^{s}(x, d)$, i.e. the solution of (2.10)-(2.12), (2.13)-(2.15) and (1.9) with $u^{i}:=e^{i k d \cdot x}$ satisfies (see [18] and [13])

$$
u^{s}(x, d)=\gamma_{m} \frac{e^{i k|x|}}{|x|^{(m-1) / 2}} u_{\infty}(\hat{x}, d)+O\left(\frac{1}{|x|}\right), \quad \hat{x}=x /|x|, \quad|x| \rightarrow \infty,
$$

where

$$
\gamma_{m}=\frac{e^{i \pi / 4}}{\sqrt{8 \pi k}} \quad \text { if } m=2 \quad \text { and } \quad \gamma_{m}=\frac{1}{4 \pi} \quad \text { if } m=3 .
$$

The function $u_{\infty}(\hat{x}, d)$ which is an analytic function of $\hat{x}$ on the unit sphere $\mathbb{S}^{m-1}:=$ $\left\{x \in \mathbb{R}^{m},|x|=1\right\}$, is referred to as the far field pattern of the scattered field $u^{s}(x, d)$.

The inverse problem we consider here is to determine the delaminated portion $\Gamma_{0}$ of the boundary $\Gamma$ from a knowledge of $u_{\infty}(\hat{x}, d)$ for $\hat{x}$ and $d$ on the unit sphere $\mathbb{S}^{m-1}$. Although in applications to nondestructive testing it is possible to have measurements all around, we remark that the inversion algorithm that we shall develop next can also be justified and implemented for limited aperture data (see Section 4.5 in [13]) as well as for near field data. However, the quality of the reconstruction is likely to be poor for small apertures which is usually the case for qualitative methods [23]. We also remark that for many problems in nondestructive testing, it is reasonable to assume that the background medium is known as we do here, since the background corresponds to the healthy object to be tested. In the cases when the background is not know and for simple defects, qualitative methods could be used to determine interfaces between homogeneous regions of the background media along with the defect (see [32] and some references therein).

4.1. A mixed reciprocity principle. We start by proving a mixed reciprocity result in order to deal with the non-homogeneous background. This generalizes similar results in [23], [9] and [16] (see also [4] for a similar type of calculations).

To this end we let $u_{b}(\cdot, d)$ be the total field due to the background, i.e. in absence of the delamination $\Gamma_{0}$, corresponding to the plain wave incident field $u^{i}(\cdot, d)$. More precisely, $u_{b}(\cdot, d)$ is the unique solution in $H_{l o c}^{1}\left(\mathbb{R}^{m}\right)$ of

$$
\begin{gathered}
\nabla \cdot\left(\frac{1}{\mu} \nabla u_{b}\right)+k^{2} n u_{b}=0 \text { in } \mathbb{R}^{m}, \\
u_{b}=u_{b}^{s}+u^{i}, \\
\lim _{r \rightarrow \infty} r^{\frac{m-1}{2}}\left(\frac{\partial u_{b}^{s}}{\partial r}-i k u_{b}^{s}\right)=0,
\end{gathered}
$$

where $\mu$ and $n$, both in $L^{\infty}(\Omega)$, are defined by (3.13). Note that the continuity of the field and co-normal derivatives across $\Gamma_{1}$ and $\Gamma$ are implicit in this formulation. Next let $G_{b}(\cdot, \cdot)$ be the Green's function associated with the background media, i.e 
$G_{b}(\cdot, z) \in H_{l o c}^{1}\left(\mathbb{R}^{m} \backslash\{z\}\right)$ satisfying

$$
\begin{gathered}
\nabla \cdot\left(\frac{1}{\mu} \nabla G_{b}(\cdot, z)\right)+k^{2} n G_{b}(\cdot, z)=-\delta(\cdot-z), \quad \text { in } \mathbb{R}^{m} \backslash\{z\}, \\
\lim _{r \rightarrow \infty} r^{\frac{m-1}{2}}\left(\frac{\partial G_{b}(\cdot, z)}{\partial r}-i k G_{b}(\cdot, z)\right)=0
\end{gathered}
$$

where again the continuity of the field and co-normal derivatives across $\Gamma_{1}$ and $\Gamma$ is understood. We denote by $G_{b}^{\infty}(\cdot, z) \in L^{2}\left(\mathbb{S}^{m-1}\right)$ the far-field pattern of the radiating field $G_{b}(\cdot, z)$.

THEOREM 4.1 (Mixed Reciprocity principle). The following relation holds

$$
G_{b}^{\infty}(\hat{x}, z)=\gamma_{m} u_{b}(z,-\hat{x}) \quad \text { for all } z \in \mathbb{R}^{m} \text { and } \hat{x} \in \mathbb{S}^{m-1},
$$

where $\gamma_{m}$ is defined by (4.1).

Proof. Let us first consider $z \in \Omega_{\mathrm{ext}}:=\mathbb{R}^{m} \backslash \bar{\Omega}$. Let $\Phi(\cdot, z)$ denote the fundamental solution of the Helmholtz equation $\Delta u+k^{2} u=0$ given by

$$
\Phi(x, z)=\left\{\begin{aligned}
\frac{i}{4} H_{0}^{(1)}(k|x-z|) & \text { in } \mathbb{R}^{2}, \\
\frac{1}{4 \pi} \frac{e^{i k|x-z|}}{|x-z|} & \text { in } \mathbb{R}^{3} .
\end{aligned}\right\}
$$

Since $G_{b}(\cdot, z)-\Phi(\cdot, z)$ is a non-singular radiating solution to $\Delta u+k^{2} u=0$ in $\Omega_{\text {ext }}$, an application of Green's second identity together with the Sommerfeld radiation condition implies that for all $x \in \Omega_{\text {ext }}$

$$
\begin{aligned}
\left(G_{b}-\Phi\right)(x, z) & =\int_{\Gamma_{1}}\left(\left(G_{b}-\Phi\right)(y, z) \frac{\partial \Phi}{\partial \nu_{y}}(x, y)-\Phi(x, y) \frac{\partial\left(G_{b}-\Phi\right)}{\partial \nu_{y}}(y, z)\right) d s_{y} \\
& =\int_{\Gamma_{1}}\left(G_{b}(y, z) \frac{\partial \Phi}{\partial \nu_{y}}(x, y)-\Phi(x, y) \frac{\partial G_{b}}{\partial \nu_{y}}(y, z)\right) d s_{y},
\end{aligned}
$$

where we have used the fact that, since $z \in \Omega_{\mathrm{ext}}$,

$$
\int_{\Gamma_{1}}\left(\Phi(y, z) \frac{\partial \Phi}{\partial \nu_{y}}(x, y)-\Phi(y, z) \frac{\partial \Phi}{\partial \nu_{y}}(x, y)\right) d s_{y}=0 .
$$

Then, from (4.5), and using the fact that $\Phi^{\infty}(\hat{x}, z)=\gamma_{m} u^{i}(z,-\hat{x}):=\gamma_{m} e^{-i \hat{x} \cdot z}$ we obtain for all $x \in \Omega_{\text {ext }}$

$$
\begin{aligned}
& G_{b}^{\infty}(\hat{x}, z)-\gamma_{m} u^{i}(z,-\hat{x})= \\
& \quad \gamma_{m} \int_{\Gamma_{1}}\left(G_{b}(y, z) \frac{\partial u^{i}}{\partial \nu_{y}}(y,-\hat{x})-u^{i}(y,-\hat{x}) \frac{\partial G_{b}}{\partial \nu_{y}}(y, z)\right) d s_{y} .
\end{aligned}
$$

On the other hand, the scattered field due to the background $u_{b}^{s}(\cdot,-\hat{x})$ is also a radiating solution of $\Delta u+k^{2} u=0$ in $\Omega_{\text {ext }}$ hence we have that

$$
\int_{\Gamma_{1}}\left(\left(\Phi-G_{b}\right)(y, z) \frac{\partial u_{b}^{s}}{\partial \nu_{y}}(y,-\hat{x})-u_{b}^{s}(y,-\hat{x}) \frac{\partial\left(\Phi-G_{b}\right)}{\partial \nu_{y}}(y, z)\right) d s_{y}=0 .
$$


Now the integral representation formula for $u_{b}^{s}(\cdot,-\hat{x})$ in $\Omega_{\text {ext }}$ (see [13]) yields

$$
\begin{aligned}
u_{b}^{s}(z,-\hat{x}) & =\int_{\Gamma_{1}}\left(u_{b}^{s}(y,-\hat{x}) \frac{\partial \Phi}{\partial \nu_{y}}(y, z)-\Phi(y, z) \frac{\partial u_{b}^{s}(y,-\hat{x})}{\partial \nu_{y}}\right) d s_{y} \\
& =\int_{\Gamma_{1}}\left(u_{b}^{s}(y,-\hat{x}) \frac{\partial G_{b}}{\partial \nu_{y}}(y, z)-G_{b}(y, z) \frac{\partial u_{b}^{s}(y,-\hat{x})}{\partial \nu_{y}}\right) d s_{y} .
\end{aligned}
$$

In addition, using the transmission conditions across the interfaces $\Gamma_{1}$ and the equations for $u_{b}$ and $G_{b}(\cdot, \cdot)$ we obtain

$$
\begin{gathered}
\int_{\Gamma_{1}}\left(u_{b}(y,-\hat{x}) \frac{\partial G_{b}}{\partial \nu_{y}}(z, y)-G_{b}(z, y) \frac{\partial u_{b}(y,-\hat{x})}{\partial \nu_{y}}\right) d s_{y} \\
=\int_{\Gamma_{1}}\left(u_{b}^{+}(y,-\hat{x}) \frac{1}{\mu^{+}} \frac{\partial G_{b}^{+}}{\partial \nu_{y}}(z, y)-G_{b}^{+}(z, y) \frac{1}{\mu^{+}} \frac{\partial u_{b}^{+}(y,-\hat{x})}{\partial \nu_{y}}\right) d s_{y} \\
=\int_{\Omega}\left(u_{b}(y,-\hat{x}) \nabla \cdot\left(\frac{1}{\mu} \nabla G_{b}\right)(z, y)-G_{b}(z, y) \nabla \cdot\left(\frac{1}{\mu} \nabla u_{b}\right)(y,-\hat{x})\right) d s_{y}=0
\end{gathered}
$$

Thus from (4.7) and (4.8), since $u_{b}=u_{b}^{s}+u^{i}$ we have that

$$
u_{b}^{s}(z,-\hat{x})=\int_{\Gamma_{1}}\left(G_{b}(z, y) \frac{\partial u^{i}(y,-\hat{x})}{\partial \nu_{y}}-u^{i}(y,-\hat{x}) \frac{\partial G_{b}}{\partial \nu_{y}}(z, y)\right) d s_{y} .
$$

Finally (4.6) provides

$$
G_{b}^{\infty}(\hat{x}, z)=\gamma_{m} u_{b}(z,-\hat{x}) .
$$

Next let $z \in \Omega_{+} \cup \Omega_{-}$. Then $G_{b}(\cdot, z)$ is a smooth radiating solution of $\Delta u+k^{2} u=0$ in $\Omega_{\text {ext }}$, and hence Green's representation formula implies

$$
G_{b}(x, z)=\int_{\Gamma_{1}}\left(G_{b}(y, z) \frac{\partial \Phi}{\partial \nu_{y}}(x, y)-\Phi_{k}(x, y) \frac{\partial G_{b}}{\partial \nu_{y}}(y, z)\right) d s_{y} .
$$

Evaluating the far field pattern yields

$$
G_{b}^{\infty}(\hat{x}, z)=\gamma_{m} \int_{\Gamma_{1}}\left(G_{b}(y, z) \frac{\partial e^{-i k \hat{x} \cdot y}}{\partial \nu_{y}}-e^{-i k \hat{x} \cdot y} \frac{\partial G_{b}(y, z)}{\partial \nu_{y}}\right) d s_{y} .
$$

Moreover, since $u_{b}^{s}(\cdot,-\hat{x})$ is also a radiating solution to the Helmholtz equation in $\Omega_{\text {ext }}$, we have that

$$
\gamma_{m} \int_{\Gamma_{1}}\left(G_{b}(y, z) \frac{\partial u_{b}^{s}(y,-\hat{x})}{\partial \nu_{y}}-u_{b}^{s}(y,-\hat{x}) \frac{\partial G_{b}(y, z)}{\partial \nu_{y}}\right) d s_{y}=0,
$$

Hence adding (4.11) and (4.12), recalling that $u_{b}(y,-\hat{x})=u_{b}^{s}(y,-\hat{x})+e^{-i k \hat{x} \cdot y}$ and applying Green's second identity and the transmission conditions across $\Gamma_{1}$ and $\Gamma$ proves that

$$
\begin{aligned}
G_{b}^{\infty}(\hat{x}, z) & =\gamma_{m} \int_{\Gamma_{1}}\left(G_{b}(y, z) \frac{\partial e^{-i k \hat{x} \cdot y}}{\partial \nu_{y}}-e^{-i k \hat{x} \cdot y} \frac{\partial G_{b}(y, z)}{\partial \nu_{y}}\right) d s_{y} \\
& =\gamma_{m} \int_{\Omega_{+} \cup \Omega_{-}}\left(G_{b}(y, z) \nabla \cdot\left(\frac{1}{\mu} \nabla u_{b}\right)(y,-\hat{x})-u_{b}(y,-\hat{x}) \nabla \cdot\left(\frac{1}{\mu} \nabla G_{b}\right)(y, z)\right) d y \\
& +\gamma_{m} \int_{\Gamma}\left(G_{b}(y, z)\left[\frac{1}{\mu} \frac{\partial u_{b}}{\partial \nu(y)}\right](y,-\hat{x})-u_{b}(y,-\hat{x})\left[\frac{1}{\mu} \frac{\partial G_{b}}{\partial \nu_{y}}\right](y, z)\right) d s_{y}
\end{aligned}
$$


Now we use the continuity of $\frac{1}{\mu} \frac{\partial u_{b}}{\partial \nu_{y}}$ and $\frac{1}{\mu} \frac{\partial G_{b}}{\partial \nu_{y}}$ across $\Gamma$ and the fact that $u_{b}$ and $G_{b}$ satisfy the same equation in $\left(\Omega_{+} \cup \Omega_{-}\right) \backslash \overline{B_{\epsilon}(z)}$, where $B_{\epsilon}(z)$ is a small ball of radius $\epsilon$ centered at $z$ and included either in $\Omega_{+}$or $\Omega_{-}$, to obtain

$G_{b}^{\infty}(\hat{x}, z)=\gamma_{m} \int_{B_{\epsilon}(z)}\left(G_{b}(y, z) \nabla \cdot\left(\frac{1}{\mu} \nabla u_{b}\right)(y,-\hat{x})-u_{b}(y,-\hat{x}) \nabla \cdot\left(\frac{1}{\mu} \nabla G_{b}\right)(y, z)\right) d y$.

Letting $\epsilon$ to zero and using the equation for $u_{b}$ and the first equation in (4.3) for $x \in B_{\epsilon}(z)$ finally implies

$$
G_{b}^{\infty}(\hat{x}, z)=\gamma_{m} u_{b}(z,-\hat{x})
$$

where we have used (4.7). Finally, by continuity of $G_{b}$ across $\Gamma_{1}$ and $\Gamma$, we can now conclude that $G_{b}^{\infty}(\hat{x}, \cdot)=\gamma_{m} u_{b}(\cdot,-\hat{x})$ holds everywhere in $\mathbb{R}^{m}$.

4.2. The linear sampling method. We now propose and analyze a version of the Linear Sampling Method (LSM) to detect the delaminated part $\Gamma_{0}$ on the known interface $\Gamma$. As mentioned earlier, the data needed for our inversion scheme is the multistatic far field pattern $u_{\infty}(\hat{x}, d), \hat{x}, d \in \mathbb{S}^{m-1}$. This far field data allows us to define the standard far field operator $F: L^{2}\left(\mathbb{S}^{m-1}\right) \rightarrow L^{2}\left(\mathbb{S}^{m-1}\right)$ given by

$$
(F g)(\hat{x})=\int_{\mathbb{S}^{m-1}} u^{\infty}(\hat{x}, d) g(d) d s_{d} .
$$

By linearity $F g$ is the far field pattern of the scattered field $u^{s}$ satisfying the scattering problem (2.10)-(2.12), (2.13)-(2.15) and (1.9) with $u^{i}:=v_{g}$, where $v_{g}$ is the so-called Herglotz wave function defined by

$$
v_{g}(x)=\int_{\mathbb{S} m-1} g(d) e^{i k x \cdot d} d s_{d} .
$$

On the other hand the far field pattern $u_{b}^{\infty}(\hat{x}, d)$ of the scattered field due to the background, i.e. the solution $u_{b}^{s}(\cdot, d)$ of (4.2), defines the background far field operator $F_{b}: L^{2}\left(\mathbb{S}^{m-1}\right) \rightarrow L^{2}\left(\mathbb{S}^{m-1}\right)$

$$
\left(F_{b} g\right)(\hat{x})=\int_{\mathbb{S}^{m-1}} u_{b}^{\infty}(\hat{x}, d) g(d) d s_{d} .
$$

Note that $F_{b} g$ can be computed since the undamaged configuration of the scatterer is known a priori. Similarly, by linearity $F_{b} g$ is the far field pattern of the solution $u_{b}^{s}$ with $u^{i}:=v_{g}$. Also by linearity, the total field $u_{b, g}$ corresponding to the scattering by the background media due to $v_{g}$ as incident field, i.e solution of (4.2) with $u^{i}:=v_{g}$, can be written as

$$
u_{b, g}(x):=\int_{\mathbb{S}^{m-1}} u_{b}(x, d) g(d) d s_{d}
$$

Finally, we define the far field operator solely due to the delamination $F_{D}: L^{2}\left(\mathbb{S}^{m-1}\right) \rightarrow$ $L^{2}\left(\mathbb{S}^{m-1}\right)$ which is given by

$$
F_{D} g=F g-F_{b} g .
$$

Obviously $F_{D} g$ can be seen as the far field pattern of the scattered field due to the defect $\Gamma_{0}$ when the incident field is $u_{b, g}$ given by (4.16). From this point we assume 
that we know $F_{D} g$, which we will use to develop the linear sampling method to reconstruct $\Gamma_{0}$. To this end, we define the bounded linear operator $H: L^{2}\left(\mathbb{S}^{m-1}\right) \rightarrow$ $H^{-1 / 2}\left(\Gamma_{0}\right) \times \mathcal{H}^{-1}\left(\Gamma_{0}\right)$ by

$$
H g=\left(\left.\alpha \frac{1}{\mu} \frac{\partial u_{b, g}}{\partial \nu}\right|_{\Gamma_{0}}, K u_{b, g}\right),
$$

where $K: \mathcal{H}\left(\Gamma_{0}\right) \rightarrow \mathcal{H}^{-1}\left(\Gamma_{0}\right)$ corresponds to one part of the boundary data on $\Gamma_{0}$ and is given by (see (3.22) and (3.24))

$$
(K \phi, \psi)_{\mathcal{H}\left(\Gamma_{0}\right), \mathcal{H}-1\left(\Gamma_{0}\right)}=\int_{\Gamma_{0}}\left\{\langle\beta f\rangle \nabla_{\Gamma} \phi \cdot \nabla_{\Gamma} \bar{\psi}+\gamma \phi \bar{\psi}\right\} d s .
$$

The conjugate transpose operator $K^{*}: \mathcal{H}\left(\Gamma_{0}\right) \rightarrow \mathcal{H}^{-1}\left(\Gamma_{0}\right)$ is defined by

$$
\left(K^{*} \phi, \psi\right)=\int_{\Gamma_{0}}\left\{\langle\bar{\beta} f\rangle \nabla_{\Gamma} \phi \cdot \nabla_{\Gamma} \bar{\psi}+\bar{\gamma} \phi \bar{\psi}\right\} d s:=(\bar{K} \phi, \psi) .
$$

Note that $H g$ maps $u_{b, g}$ to the corresponding transmission conditions given by (3.22), since both the field $u_{b, g}$ and its co-normal derivative are continuous on $\Gamma_{0}$ (so the terms in (3.22) with jumps disappear) and we simply write the average by the common value on either side of the curve, i.e $\left\langle\frac{1}{\mu} \frac{\partial u_{b, g}}{\partial \nu}\right\rangle=\frac{1}{\mu^{ \pm}} \frac{\partial u_{b, g}^{ \pm}}{\partial \nu}$, and $\left\langle u_{b, g}\right\rangle=u_{b, g}^{ \pm}$. We remark that for smooth $\Gamma_{0}$ and smooth coefficients $\mu^{ \pm}$and $n^{ \pm}$, we can assume by the regularity of the solution of the transmission problem that $u_{b, g} \in \mathcal{H}$ and hence its trace on $\Gamma_{0}$ is in $\mathcal{H}\left(\Gamma_{0}\right)$.

LEMmA 4.2. The operator $H: L^{2}\left(\mathbb{S}^{m-1}\right) \rightarrow H^{-1 / 2}\left(\Gamma_{0}\right) \times \mathcal{H}^{-1}\left(\Gamma_{0}\right)$ has dense range. Assume in addition to the assumptions of Theorem 3.3 that $\Re\left(n-n_{0}\right)>0$ (or more generally that there is no non-trivial $u_{b, g}$ such that $K u_{b, g}=0$ ), then $H$ is injective.

Proof. We first check the injectivity. Let $g \in L^{2}\left(\mathbb{S}^{m-1}\right)$ such that $H g=0$. Then both $\left.\frac{1}{\mu} \frac{\partial u_{b, g}}{\partial \nu}\right|_{\Gamma_{0}}$ and $\left.u_{b, g}\right|_{\Gamma_{0}}=0$. The latter follows by taking the real part of $K u_{b, g}=0$ and the fact that $\Re(\langle\beta f\rangle)>0$ and $\Re(\gamma)>0$. Then, by Holmgren's theorem we conclude that $u_{b, g}=0$ in a region extending on both sides of $\Gamma_{0}$, and by analytic continuation we obtain that $u_{b, g} \equiv 0$ vanishes identically. Since $u_{b, g}$ is sum of radiating scattering wave and the Herglotz wave function $v_{g}$ which is an entire solution to Helmholtz equation, the latter implies $v_{g} \equiv 0$ yielding $g=0$. Next, to show that $H$ has dense range it suffices to prove that $H^{*}$ is injective, where $H^{*}: \tilde{H}^{1 / 2}\left(\Gamma_{0}\right) \times \mathcal{H}\left(\Gamma_{0}\right) \rightarrow L^{2}\left(\mathbb{S}^{m-1}\right)$ is the transpose-conjugate operator associated with $H$. To this end, suppose that $(\zeta, \eta)$ in $\tilde{H}^{1 / 2}\left(\Gamma_{0}\right) \times \mathcal{H}\left(\Gamma_{0}\right)$. Then

$$
\begin{aligned}
(H g,(\zeta, \eta)) & =\left(\frac{\alpha}{\mu} \frac{\partial u_{b, g}}{\partial \nu}, \zeta\right)+\left(K u_{b, g}, \eta\right)=\left(\frac{\alpha}{\mu} \frac{\partial u_{b, g}}{\partial \nu}, \zeta\right)+\left(u_{b, g}, \bar{K} \eta\right) \\
& =\int_{\Gamma_{0}}\left(\frac{\alpha}{\mu} \frac{\partial u_{b, g}}{\partial \nu_{y}} \bar{\zeta}+u_{b, g} K \bar{\eta} \mathrm{d} s_{y}\right) \\
& =\int_{\mathbb{S}^{m-1}} g(\hat{x}) \int_{\Gamma_{0}}\left(\bar{\zeta} \frac{\alpha}{\mu} \frac{\partial u_{b}(y, \hat{x})}{\partial \nu_{y}}+K \bar{\eta} u_{b}(y, \hat{x})\right) \mathrm{d} s_{y} \mathrm{~d} s_{\hat{x}} \\
& =\left(g, H^{*}(\zeta, \eta)\right) .
\end{aligned}
$$

Thus

$$
H^{*}(\zeta, \eta)=\int_{\Gamma_{0}}\left(\frac{\bar{\alpha} \mu}{\bar{\mu}} \frac{1}{\mu} \frac{\partial u_{b}(y,-\hat{x})}{\partial \nu_{y}} \zeta+u_{b}(y,-\hat{x}) \bar{K} \eta\right) \mathrm{d} s_{y} .
$$


From the mixed reciprocity relation Theorem 4.1, we have that that $H^{*}(\zeta, \eta)$ is the far field pattern associated with the scattered wave

$$
w^{s}(x)=\gamma_{m}^{-1} \int_{\Gamma_{0}}\left(\zeta \frac{\bar{\alpha} \mu}{\bar{\mu}} \frac{1}{\mu} \frac{\partial G_{b}(x, y)}{\partial \nu_{y}}+\bar{K} \eta G_{b}(x, y)\right) \mathrm{d} s_{y}
$$

where $\gamma_{m}$ is defined in (4.1). Moreover, since the singularity of the free space Green's function $G_{b}(\cdot, \cdot)$ is of the same order as the fundamental solution $\Phi(\cdot, \cdot), w^{s}$ assumes following representation formula (see e.g. [28])

$$
w^{s}(x)=\int_{\Gamma_{0}}\left(\left[w^{s}\right] \frac{1}{\mu} \frac{\partial G_{b}(x, y)}{\partial \nu_{y}}-\left[\frac{1}{\mu} \frac{\partial w^{s}}{\partial \nu}\right] G_{b}(x, y)\right) \mathrm{d} s_{y},
$$

and thus

$$
\left[w^{s}\right]=\gamma_{m}^{-1} \frac{\bar{\alpha} \mu}{\bar{\mu}} \zeta \quad \text { and } \quad\left[\frac{1}{\mu} \frac{\partial w^{s}}{\partial \nu}\right]=-\gamma_{m}^{-1} \bar{K} \eta .
$$

Therefore, if $H^{*}(\zeta, \eta)=0$, then by Rellich's lemma together with the unique continuation principle and Holmgren's theorem, $w^{s}=0$ in $\mathbb{R}^{m} \backslash \bar{\Gamma}_{0}$, so $\left[w^{s}\right]=0$ and $\left[\frac{1}{\mu} \frac{\partial w^{s}}{\partial \nu}\right]=0$, implying that $\zeta=\eta=0$. by

Next, define the bounded linear operator $G: H^{-1 / 2}\left(\Gamma_{0}\right) \times \mathcal{H}^{-1}\left(\Gamma_{0}\right) \rightarrow L^{2}\left(\mathbb{S}^{m-1}\right)$

$$
G:\left(h_{1}, h_{2}\right) \mapsto w^{\infty}
$$

where $w^{\infty}$ is the far field pattern of the corresponding radiating solution $w$ to (3.18)(3.21). Notice here that the the well-posedness of the problem guarantees that the operator $G$ is well defined and bounded, since in the variational formulation the source terms $h_{1}, h_{2}$ always define a bounded linear functional in the space $\mathcal{H}$. It is clear from the definition of $H$ and $G$ that we have the factorization $F_{D}=G H$.

Since for our inverse problem we know the interface $\Gamma$ and are looking for the delaminated part $\Gamma_{0}$, we define the test function as follows: For any $L \subset \Gamma$, given $\left(\alpha_{L}, \beta_{L}\right) \in L^{2}(L) \times \tilde{H}^{1}(L)$ we define

$$
\phi_{L}^{\infty}(\hat{x}):=\gamma_{m} \int_{L}\left\{\alpha_{L}(y) u_{b}(y,-\hat{x})+\beta_{L}(y) \frac{1}{\mu} \frac{\partial u_{b}(y,-\hat{x})}{\partial \nu(y)}\right\} d s(y)
$$

where $\hat{x}=x /|x|$. Then, we can prove the following

Lemma 4.3. Let $L \subset \Gamma$ and $\left(\alpha_{L}, \beta_{L}\right) \in L^{2}(L) \times \tilde{H}^{1}(L)$, not simultaneously zero. Then $L \subset \Gamma_{0}$ if and only if $\phi_{L}^{\infty} \in \operatorname{Range}(G)$.

Proof. Let's first assume that $L \subset \Gamma_{0}$. Then the corresponding extensions by zero in $\Gamma_{0},\left(\tilde{\alpha}_{L}, \tilde{\beta}_{L}\right)$, are in $L^{2}\left(\Gamma_{0}\right) \times \tilde{H}^{1}\left(\Gamma_{0}\right)$, and the potential

$$
\phi_{0}(x):=\int_{\Gamma_{0}}\left\{\tilde{\alpha}_{L}(y) G_{b}(x, y)+\tilde{\beta}_{L}(y) \frac{1}{\mu} \frac{\partial G_{b}(x, y)}{\partial \nu(y)}\right\} d s(y)
$$

belongs to $H_{l o c}^{1}\left(\mathbb{R}^{m} \backslash \bar{\Gamma}_{0}\right)$ and satisfies

$$
\left[\phi_{0}\right]=\tilde{\beta}_{L}, \quad\left[\frac{1}{\mu} \frac{\partial \phi_{0}}{\partial \nu}\right]=-\tilde{\alpha}_{L} \quad \text { on } \quad \Gamma_{0} .
$$


Let's now denote by $\mathbf{S}_{\Gamma_{0}}$ and $\mathbf{K}_{\Gamma_{0}}$ the restriction to $\Gamma_{0}$ of the generalized single and double layer potentials, defined by

$$
\left(\mathbf{S}_{\Gamma_{0}} \psi\right)(x):=\int_{\Gamma_{0}} \psi(y) G_{b}(x, y) d s(y), \quad x \in \Gamma_{0}
$$

and

$$
\left(\mathbf{K}_{\Gamma_{0}} \psi\right)(x):=\int_{\Gamma_{0}} \psi(y) \frac{\partial}{\partial \nu(y)} G_{b}(x, y) d s(y), \quad x \in \Gamma_{0} .
$$

In [13], it is shown that $\mathbf{S}_{\Gamma_{0}}: \tilde{H}^{-\frac{1}{2}+s}\left(\Gamma_{0}\right) \rightarrow H^{\frac{1}{2}+s}\left(\Gamma_{0}\right)$ and $\mathbf{K}_{\Gamma_{0}}: \tilde{H}^{\frac{1}{2}+s}\left(\Gamma_{0}\right) \rightarrow$ $H^{\frac{1}{2}+s}\left(\Gamma_{0}\right)$ are continuous for every $-1 \leq s \leq 1$ (here $\tilde{H}^{r}\left(\Gamma_{0}\right)$ denotes the space of functions that can be extended by zero to the whole $\Gamma$ as functions in $H^{r}(\Gamma)$ ). Since, by the transmission conditions (4.23), we know that $\left[\frac{1}{\mu} \frac{\partial \phi_{0}}{\partial \nu}\right] \in L^{2}\left(\Gamma_{0}\right)$ and $\left[\phi_{0}\right] \in \tilde{H}^{1}\left(\Gamma_{0}\right)$, together with the fact that $\left\langle\phi_{0}\right\rangle=-\mathbf{S}_{\Gamma_{0}}\left[\frac{1}{\mu} \frac{\partial \phi_{0}}{\partial \nu}\right]+\mathbf{K}_{\Gamma_{0}}\left[\phi_{0}\right]$, we have that $\left\langle\phi_{0}\right\rangle \in H^{1}\left(\Gamma_{0}\right)$ and hence the potential $\phi_{0}$ belongs to $\mathcal{H}$. Therefore, $\phi_{0}$ satisfies (3.18)-(3.21) with $h_{1}$ and $h_{2}$ defined by (3.22) for $v=-\phi_{0} \in \mathcal{H}$, implying that $G\left(h_{1}, h_{2}\right)=\phi_{L}^{\infty}$. To prove the converse, let's suppose that $L \not \subset \Gamma_{0}$ but that there exists a pair $\left(\alpha_{L}, \beta_{L}\right) \in L^{2}(L) \times \tilde{H}^{1}(L)$, not simultaneously zero, such that $\phi_{L}^{\infty} \in \operatorname{Range}(G)$. By definition of $G$, there exists $\left(h_{1}, h_{2}\right)$ in $H^{-1 / 2}\left(\Gamma_{0}\right) \times \mathcal{H}\left(\Gamma_{0}\right)$ such that $\phi_{L}^{\infty}=w^{\infty}$, where $w$ satisfies (3.18)-(3.21). Therefore, $\phi_{L}^{\infty}$ is the far field pattern of the two potentials:

$$
\phi_{L}(x)=\gamma_{m}^{-1} \int_{L}\left\{\alpha_{L}(y) G_{b}(x, y)+\beta_{L}(y) \frac{1}{\mu} \frac{\partial G_{b}(x, y)}{\partial \nu(y)}\right\} d s(y)
$$

and

$$
w(x)=\int_{\Gamma_{0}}\left\{\left[\frac{1}{\mu} \frac{\partial w}{\partial \nu(y)}\right](y) G_{b}(x, y)+[w](y) \frac{1}{\mu} \frac{\partial G_{b}(x, y)}{\partial \nu(y)}\right\} d s(y) .
$$

By Rellich's lemma, unique continuation, and Holmgren's theorem, $w=\phi_{L}$ identically in $\mathbb{R}^{m} \backslash \overline{\Gamma_{0} \cup L}$. However this is a contradiction, because given for example any point $x_{0} \in L \backslash \bar{\Gamma}_{0}$, both $w$ and the co-normal derivative $\frac{1}{\mu} \frac{\partial w}{\partial \nu_{L}}$ are continuous at $x_{0}$, whereas either $\phi_{L}$ or the co-normal derivative $\frac{1}{\mu} \frac{\partial \phi_{L}}{\partial \nu_{L}}$ have a jump across $L$ at $x_{0}$ (since either $\alpha_{L}$ or $\beta_{L}$ doesn't vanish at that point).

Lemma 4.4. Assume in addition to the assumptions of Theorem 3.3 that $\Re(n-$ $\left.n_{0}\right)>0$ (or more generally there is no non-trivial $u_{b, g}$ such that $K u_{b, g}=0$ ). Then $F_{D}: L^{2}\left(\mathbb{S}^{m-1}\right) \rightarrow L^{2}\left(\mathbb{S}^{m-1}\right)$ is injective and has dense range.

Proof. Since $F_{D}=G H$, the injectivity follows from Lemma 4.2 and the fact that the operator $G$ is injective due to the well-posedness of (3.18)-(3.21). Next, since the range of $H$ is dense in $H^{-1 / 2}\left(\Gamma_{0}\right) \times \mathcal{H}^{-1}\left(\Gamma_{0}\right)$ it suffices to show that the range of $G$ is dense. From Lemma 4.3, in particular we have that functions $P \psi$ of the form

$$
(P \psi)(\hat{x}):=\int_{\Gamma_{0}} \psi(y) u_{b}(y,-\hat{x}) d y=\gamma_{m}^{-1} \int_{\Gamma_{0}} \psi(y) G_{b}^{\infty}(\hat{x}, y) d y
$$

are in the range of $G$ for all $\psi \in L^{2}\left(\Gamma_{0}\right)$. The set $\left\{P \psi\right.$ for all $\left.\psi \in L^{2}\left(\Gamma_{0}\right)\right\}$ is dense in $L^{2}\left(\mathbb{S}^{m-1}\right)$. Indeed, let us consider $P: L^{2}\left(\Gamma_{0}\right) \rightarrow L^{2}\left(\mathbb{S}^{m-1}\right)$. Its adjoint $P^{*}$ : $L^{2}\left(\mathbb{S}^{m-1}\right) \rightarrow L^{2}\left(\Gamma_{0}\right)$ is given by

$$
\left(P^{*} g\right)(y)=\int_{\mathbb{S}^{m-1}} g(\hat{x}) \overline{u_{b}(y,-\hat{x})} d \hat{x}=\overline{u_{b, h}}(y)
$$


where $h(\hat{x}):=\bar{g}(-\hat{x})$ and $u_{b, h}$ is given by (4.16). Now the total field due to the background medium $u_{b, h}$ corresponding to the Herglotz wave function $v_{h}$ as incident wave can not be zero unless $h=0$, since it is sum of an outgoing wave (scattered field) and incoming wave (the incident wave). This implies that $P^{*}$ is injective which finishes the proof.

Now we are ready to characterize $\Gamma_{0}$ in terms of the behavior of the approximate solution to the far-field equation

$$
F_{D} g=\phi_{L}^{\infty}
$$

The following main theorem is a summary of the all the above results.

Theorem 4.5 (Linear Sampling Method). Let $F_{D}: L^{2}\left(\mathbb{S}^{m-1}\right) \rightarrow L^{2}\left(\mathbb{S}^{m-1}\right)$ be the far field operator corresponding given by (4.17). Then:

1. For an arbitrary arc $L \subset \Gamma_{0}$ and $\epsilon>0$, there exists a function $g_{L}^{\epsilon} \in L^{2}\left(\mathbb{S}^{m-1}\right)$ such that

$$
\left\|F_{D} g_{L}^{\epsilon}-\phi_{\infty}^{L}\right\|_{L^{2}\left(\mathbb{S}^{m-1}\right)}<\epsilon
$$

and, as $\epsilon \rightarrow 0$, the corresponding solution $u_{b, g_{L}^{\epsilon}}$ to the background problem (4.2) converges in $\mathcal{H}$ to the unique solution $u_{L}$ of (3.18)-(3.21) with $h_{1}=$ $\alpha\left\langle\frac{1}{\mu} \frac{\partial \phi_{L}^{\infty}}{\partial \nu}\right\rangle$ and $h_{2}=K\left\langle\phi_{L}^{\infty}\right\rangle$ on $\Gamma_{0}$.

2. For $L \not \subset \Gamma_{0}$ and $\epsilon>0$, every function $g_{L}^{\epsilon} \in L^{2}\left(\mathbb{S}^{m-1}\right)$ such that

$$
\left\|F_{D} g_{L}^{\epsilon}-\phi_{\infty}^{L}\right\|_{L^{2}\left(\mathbb{S}^{m-1}\right)}<\epsilon
$$

is such that the corresponding solution $u_{b, g_{L}^{\epsilon}}$ to the background problem (4.2) satisfies

$$
\lim _{\epsilon \rightarrow 0}\left\|u_{b, g_{L}^{\epsilon}}\right\|_{\mathcal{H}}=\infty \quad \text { and } \quad \lim _{\epsilon \rightarrow 0}\left\|g_{L}^{\epsilon}\right\|_{L^{2}\left(\mathbb{S}^{m-1}\right)}=\infty .
$$

This theorem constitute the foundation of the linear sampling method which we will implement in the next section.

5. Numerical examples for the inverse problem. In this section we show how the linear sampling method that we have just developed can be applied numerically, and show its viability by some numerical examples. From the statement of Theorem 4.5, we know that the approximate solution of the far-field equation $F_{D} \tilde{g}_{L}=\phi_{L}^{\infty}$ can be used to detect the delaminated part $\Gamma_{0}$. Unfortunately, the far field equation is ill-posed since the far-field operator $F_{D}$ is compact, and of course the discrete counterpart, $A g_{L}=f_{L}$, will inherit the ill-posedness as ill-conditioning. Therefore, it has to be solved by means of a regularization method.

Let us first discuss the construction of the discrete far-field operator $A$ and the right hand side $f_{L}$. In all the numerical examples that we present in this section, the discrete counterpart of the far-field operator is the matrix $A \in \mathbb{C}^{40 \times 40}$, such that $A_{i j}=u^{\infty}\left(\hat{x}_{i}, \hat{d}_{j}\right)-u_{b}^{\infty}\left(\hat{x}_{i}, \hat{d}_{j}\right)$, where $u^{\infty}\left(\cdot, \hat{d}_{j}\right)$ and $u_{b}^{\infty}\left(\cdot, \hat{d}_{j}\right)$ are the the far-field pattern of the scattering problem with and without delamination, respectively, when the incident one is $u^{i n c}\left(x, \hat{d}_{j}\right)=e^{i k x \cdot \hat{d}_{j}}$. Here we take $\hat{d}_{j}=(\cos (2 \pi j / 40), \sin (2 \pi j / 40))$, and $\hat{x}_{i}=(\cos (2 \pi i / 40), \sin (2 \pi i / 40))$, for $i, j=0,1, \ldots, 39$. In order to see the stability of the reconstruction method with respect to noise, we added some random noise to the computed far field for the approximate crack problem, so we actually consider $\tilde{A}_{i j}=A_{i j}\left(1+\epsilon \xi_{i j}\right)$, where $\left\{\xi_{i j}\right\}$ is a collection of independent random variables with 
uniform distribution over the interval $[-0.5,0.5]$, and $\epsilon>0$ is a constant chosen so that the relative noise $\rho:=\|A-\tilde{A}\|_{2} /\|A\|_{2}$ attains the desired value. In each example $\rho$ is computed and specified.

Since $f_{L}$ is the discrete version of the right hand side of equation (4.24) and we have some freedom to choose the densities $\alpha_{L}$ and $\beta_{L}$, we decided to consider $\alpha_{L}$ as an approximation of $\delta_{z}$ (where $\delta_{z}$ is the Dirac delta located on $z \in \Gamma$ ) and $\beta_{L}=0$. Then, for a given finite set of points $\left\{z_{j}\right\} \subset \Gamma$, our discrete right hand side simplifies to

$$
\left(f_{z_{j}}\right)_{k}=u_{b}\left(z_{j},-\hat{d}_{k}\right) .
$$

Since $\Gamma$ is already known there are many other possibilities for choosing the sampling $\operatorname{arc} L$ and test functions $\alpha_{L}, \beta_{L}$ but we have not tried them here. Nevertheless, as the numerical examples show, our choice give reasonable reconstructions. In all the numerical examples that we present, we chose a collection of equally distributed points along the interface $\Gamma,\left\{z_{k}\right\}_{k=1}^{64}$. In order to "solve" each of the 64 ill-conditioned linear equations

$$
\tilde{A}_{\rho} g_{k}=f_{z_{k}},
$$

we use the well-known Tikhonov regularization method, that consists in solving the following minimization problems instead

$$
g_{k}^{\lambda}=\operatorname{argmin}_{g \in \mathbb{C}^{40}}\left\{\left\|\tilde{A}_{\rho} g-f_{z_{k}}\right\|^{2}+\lambda\|g\|^{2}\right\},
$$

where the regularization parameter was arbitrarily chosen as $\lambda=10^{-10}$. The solution of these problems was made using the free Matlab package regtools (see [25]).

As stated in Theorem (4.5), the value of $\left\|g_{k}^{\lambda}\right\|^{-1}$ is large if $z_{k}$ is in the crack support $\Gamma_{0}$, and small otherwise. Therefore, it can be used to identify the location of $\Gamma_{0}$. In the reconstructions that we present, we show results for four different noise levels $\rho$, in three different settings (a circle with one single crack, a kite with one single crack, and a kite with two cracks). For visualization purposes, in our reconstructions the separation of the dotted lines $\tilde{\Gamma}_{ \pm}$is chosen to be proportional to $\Theta\left(z_{k}\right)=\left\|g_{k}^{\lambda}\right\|^{-1}$, with the parametrization:

$$
\chi_{\tilde{\Gamma}_{ \pm}}(t)=\chi_{\Gamma}(t) \pm \eta_{*} \Theta\left(\chi_{\Gamma}(t)\right) \nu(t)
$$

where $\chi_{\Gamma}$ is the parametrization of $\Gamma$, and we arbitrarily set $\eta_{*}=0.04$ as a constant that modulates the size of $\Theta$ for pure visualization purposes. The openings of the dotted lines $\tilde{\Gamma}_{ \pm}$correspond, therefore, to the predicted location of the cracks by the linear sampling method just developed in section 4. All the numerical experiments presented here were made for layered obstacles with parameters $n_{-}=4, n_{+}=2$, $\mu_{-}=\mu_{+}=1, \mu_{0}=0.9, n_{0}=0.2$, and wave number $k=3$. Numerical examples are presented in Figure 7, Figure 8 and Figure 9 indicate that our reconstruction method provides reasonable reconstructions of $\Gamma_{0}$ even in the presence of noise.

Conclusion. We have derived a asymptotic model for the delamination of a two materials that successfully approximates scattering from thin delaminated regions. This model was shown to be well-posed and was then used to derive a new inverse scattering technique based on a modified linear sampling method that we showed can detect delamination in model problems. We note that the asymptotic model has an independent interest and could be the basis for applying other inversion techniques such back-propagation techniques or Kirchhoff migration. 

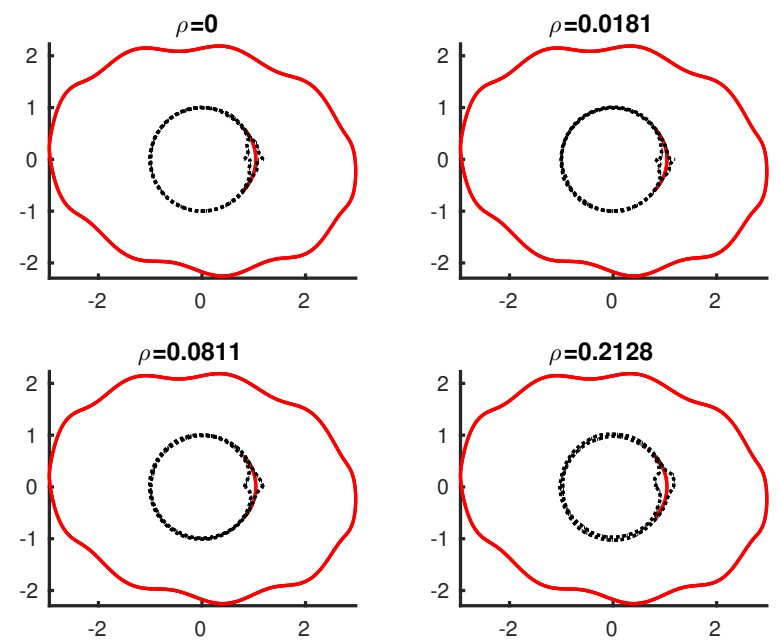

FIG. 7. Reconstruction of a single crack $\Gamma_{0}$ in a circular interface, for four levels of noise $\rho$. The solid line at the circular interface is the exact location of the crack, and the opening between the dotted lines $\chi_{\tilde{\Gamma}_{ \pm}}$is the predicted location of $\Gamma_{0}$. The outer lighter coloured curve is $\Gamma_{1}$.
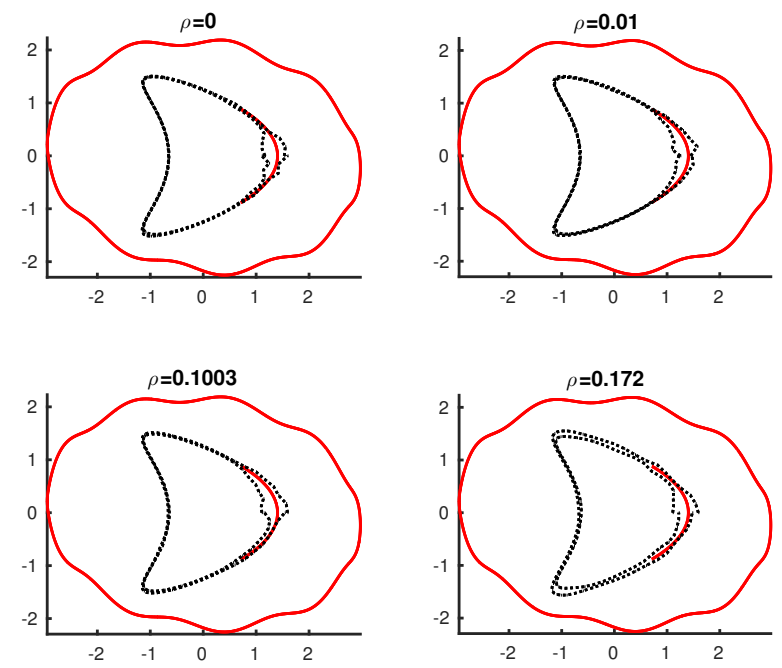

FIG. 8. Reconstruction of a single crack $\Gamma_{0}$ in a kite-shaped interface of a two-layered media, for four levels of noise $\rho$. The solid line at the kite-shaped interface is the exact location of the crack, and the opening between the dotted lines $\chi_{\tilde{\Gamma}_{ \pm}}$is the predicted location of $\Gamma_{0}$. The outer lighter colored curve is $\Gamma_{1}$.

A stability and resolution analysis of the inverse method has yet to be undertaken.

Our study raises the interesting theoretical question of proving convergence of the asymptotic asymptotic model as the thickness of the delamination goes to zero. 

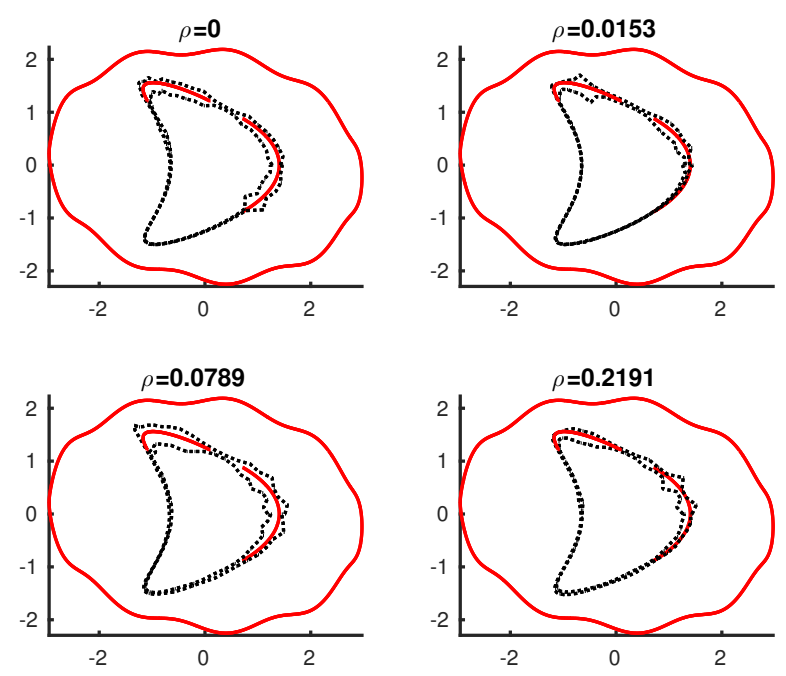

FIG. 9. Reconstruction of two cracks $\Gamma_{0}^{1} \cup \Gamma_{0}^{2}$ in a kite-shaped interface of a two-layered media for four levels of noise $\rho$. The solid line at the kite-shaped interface is the exact location of the crack, and the opening between the dotted lines $\chi_{\tilde{\Gamma}_{ \pm}}$is the predicted location of $\Gamma_{0}$. The outer lighter colored curve is $\Gamma_{1}$.

It would also be desirable to test the problem in three dimensions. Extensions to Maxwell's equations and elasticity are challenging, but are currently underway.

Acknowledgments. The research of F. Cakoni is supported in parts by AFOSR grant FA9550-13-1-0199 and NSF Grant DMS-1602802. The research of I. de Teresa is supported in part by NSF Grant DMS-1602802 the DRI of INRIA (Associate Team ISIP). I. de Teresa gratefully acknowledges the hospitality of the DeFI Team during her visit at Ecole Polytechnique. The research of P. Monk is supported in part by AFOSR grant FA9550-13-1-0199.

\section{References.}

[1] R. Adams. Sobolev spaces. Elsevier, 2nd edition, 2003.

[2] H. Ammari, E. Beretta, E. Francini, H. Kang, and M. Lim. Optimization algorithm for reconstructing interface changes of a conductivity inclusion from modal measurements. Math. Comp., 79:1757-1777, 2010.

[3] H. Ammari, E. Beretta, E. Francini, H. Kang, and M. Lim. Reconstruction of small interface changes of an inclusion from modal measurements II: the elastic case. J. Math. Pures Appl. (9), 94:322-339, 2010.

[4] H. Ammari, J. Garnier, H. Kang, M. Lim, and K. Sølna. Multistatic imaging of extended targets. SIAM J. Imaging Sci., 5:564-600, 2012.

[5] H. Ammari and S. He. Effective impedance boundary conditions for an inhomogeneous thin layer on a curved metallic surface. IEEE Transactions on Antennas and Propagation, 46:710-716, 1998.

[6] H. Ammari, H. Kang, E. Kim, K. Louati, and M. Vogelius. A MUSIC-type algorithm for detecting internal corrosion from electrostatic boundary measurements. Numer. Math., 108:501-528, 2008.

[7] B. Aslanyürek, H. Haddar, and H. Şahintürk. Generalized impedance boundary 
conditions for thin dielectric coatings with variable thickness. Wave Motion, 48:681-700, 2011.

[8] F. Ben Hassen, Y. Boukari, and H. Haddar. Application of the linear sampling method to identify cracks with impedance boundary conditions. Inverse Probl. Sci. Eng., 21:210-234, 2013.

[9] O. Bondarenko, A. Kirsch, and X. Liu. The factorization method for inverse acoustic scattering in a layered medium. Inverse Problems, 29, 2013. Art. No. 045010.

[10] Y. Boukari and H. Haddar. The factorization method applied to cracks with impedance boundary conditions. Inverse Problems and Imaging, 7:1123-1138, 2013.

[11] G. Briotti and C. Scarponi. Acoustic attenuation for ultrasonic ndi detection of delaminations on composite laminates. Journal of Reinforced Plastics and Composites, 20:176-187, 2001.

[12] F. Cakoni and D. Colton. The linear sampling method for cracks. Inverse Problems, 19:279-295, 2003.

[13] F. Cakoni and D. Colton. A Qualitative Approach to Inverse Scattering Theory. Springer, 2014.

[14] F. Cakoni, D. Colton, and H. Haddar. Transmission eigenvalues and inverse scattering theory. To appear.

[15] F. Cakoni, D. Colton, and P. Monk. The Linear Sampling Method in Inverse Electromagnetic Scattering. CBMS-NSF Regional Conference Series in Applied Mathematics. SIAM, 2011.

[16] F. Cakoni and I. Harris. The factorization method for a defective region in an anisotropic material. Inverse Problems, 31, 2015. Art. No. 025002.

[17] M. Chamaillard, N. Chaulet, and H. Haddar. Analysis of the factorization method for a general class of boundary conditions. J. Inverse Ill-Posed Problems, 22:643670, 2014.

[18] D. Colton and R. Kress. Inverse Acoustic and Electromagnetic Scattering Theory. Springer-Verlag, 3rd edition, 2013.

[19] I. de Teresa. Asymptotic Methods in Inverse Scattering for Inhomogeneous Media. $\mathrm{PhD}$ thesis, University of Delaware, 2016. In preparation.

[20] B. Delourme. Modeles et asymptotiques des interfaces fines et periodiques en electromagnetisme. PhD thesis, Universite Pierre et Marie Curie - Paris VI, 2010. In French.

[21] B. Delourme, H. Haddar, and P. Joly. Approximate models for wave propagation across thin periodic interfaces. J. Math. Pures Appl., 98:28-71, 2012.

[22] H. Gao, S. Ali, and B. López. Efficient detection of delamination in multilayered structures using ultrasonic guided wave. EMATs, NDT \& E International, 43:316-322, 2010.

[23] Y. Grisel, V. Mouysset, P. A. Mazet, and J. P. Raymond. Determining the shape of defects in non-absorbing inhomogeneous media from far-field measurements. Inverse Problems, 28, 2012. Art. No. 055003.

[24] H. Haddar, P. Joly, and H. M. Nguyen. Generalized impedance boundary conditions for scattering by strongly absorbing obstacles: the scalar case. Mathematical Models and Methods in Applied Sciences, 18:1273-1300, 2005.

[25] P.C. Hansen. http://www. mathworks .com/matlabcentral/fileexchange/52-regtools.

[26] L. Hörmander. The Analysis of Linear Partial Differential Operators III. Springer, 1985. 
[27] A. Kirsch and S. Ritter. A linear sampling method for inverse scattering from an open arc. Inverse Problems, 16:89-105, 2000.

[28] W. McLean. Strongly elliptic systems and boundary integral equations. Cambridge University Press, 2000.

[29] Ö. Özdemir, H. Haddar, and A. Yaka. Reconstruction of the electromagnetic field in layered media using the concept of approximate transmission conditions. IEEE Transactions on Antennas and Propagation, 59:2964-2972, 2011.

[30] P. Shokouhi, J. Wolf, and H. Wiggenhauser. Detection of delamination in concrete bridge decks by joint amplitude and phase analysis of ultrasonic array measurements. Journal of Bridge Engineering, 19, 2014. Art. No. 04013005.

[31] C. Soutis and S. H. Díaz Valdés. Delamination detection in laminated composites using lamb waves. In E. E. Gdoutos and Z. P. Marioli-Riga, editors, Recent Advances in Composite Materials: In Honor of S.A. Paipetis, pages 109-126. Springer Netherlands, 2003.

[32] J. Yang, B. Zhang, and H. Zhang. The factorization method for reconstructing a penetrable obstacle with unknown buried objects. SIAM J. Appl. Math., 73:617635,2013 .

[33] N. Zeev and F. Cakoni. The identification of thin dielectric objects from far field or near field scattering data. SIAM J. Appl. Math., 69:1024-1042, 2009. 